\title{
KMS and ground states on ultragraph $\mathrm{C}^{*}$-algebras
}

\author{
November 16, 2018 \\ Gilles Gonçalves de Castro and Daniel Gonçalvest 1
}

\begin{abstract}
We describe KMS and ground states arising from a generalized gauge action on ultragraph $\mathrm{C}^{*}$-algebras. We focus on ultragraphs that satisfy Condition (RFUM), so that we can use the partial crossed product description of ultragraph $\mathrm{C}^{*}$-algebras recently described by the second author and Danilo Royer. In particular, for ultragraphs with no sinks, we generalize a recent result by Toke Carlsen and Nadia Larsen: Given a time evolution on the $\mathrm{C}^{*}$-algebra of an ultragraph, induced by a function on the edge set, we characterize the KMS states in five different ways and ground states in four different ways. In both cases we include a characterization given by maps on the set of generalized vertices of the ultragraph. We apply this last result to show the existence of KMS and ground states for the ultragraph $\mathrm{C}^{*}$-algebra that is neither an Exel-Laca nor a graph $\mathrm{C}^{*}$-algebra.
\end{abstract}

Keywords: KMS states, ultragraph $\mathrm{C}^{*}$-algebras, partial crossed product.

MSC2010: 46L30, 46L55.

\footnotetext{
${ }^{1}$ Partially supported by Conselho Nacional de Desenvolvimento Científico e Tecnológico $(\mathrm{CNPq})$ - Brazil
} 


\section{Introduction}

KMS (Kubo-Martin-Schwinger) states on $\mathrm{C}^{*}$-algebras have been the subject of intense research both in Mathematics and Physics. The mathematical study of KMS states stemmed from the realization that in the $\mathrm{C}^{*}$-algebraic formulation of quantum statistical mechanics, KMS states are equilibrium states associated to the one-parameter group of automorphisms of time evolution (see [5]). Over the years a large literature was developed, as researches studied KMS states on $\mathrm{C}^{*}$-algebras associated to expansive maps (see [17]), graphs (see [15, 7]), relative graphs (see [6]), higher rank graphs (see [3]), local homemorphisms (see [1]), and Fell bundles over groupoids (see [2]), to name a few. It is interesting to note that, as mentioned in [2], KMS states make sense for any $\mathrm{C}^{*}$-dynamical system, and there is significant evidence that the KMS data is a useful invariant of a dynamical system. For example, the results in [9] show that the KMS data for a Cuntz-Krieger algebra encodes the topological entropy of the associated shift space.

Ultragraphs are generalizations of graphs, where the range map take values on the power set of the vertices. Ultragraph $\mathrm{C}^{*}$-algebras were introduced by Tomforde in [22] and they were key in the study of the relations between Exel-Laca and graph algebras (see [16]), provided new examples of algebras associated to combinatorial data (see [14], [21]), and are connected to the theory of the Perron-Frobenious operator (see [11]). Recently ultragraphs and their $\mathrm{C}^{*}$-algebras have played an important role in the study of shift spaces associated to infinite alphabets, see [12, 13]. In particular, based on ultragraph $\mathrm{C}^{*}$-algebra theory, in [13] a new approach to shifts of finite type over infinite alphabets was proposed.

Given the above mentioned relation between KMS data and topological entropy of shifts, it is relevant to study KMS states associated to ultragraph $\mathrm{C}^{*}$-algebras. This will be the focus of this article. In our main theorem (see Theorem 4.3), given an ultragraph $\mathcal{G}$ with no sinks that satisfy Condition (RFUM), we identify the KMS states on $C^{*}(\mathcal{G})$ with a subset of the states on a Abelian algebra $(C(X))$, a subset of the regular Borel probability measures on $X$, a subset of the functions on the generalized vertices of $\mathcal{G}$, and 
a subset of the states on the core subalgebra of $C^{*}(\mathcal{G})$. Taking advantage of the results proved to characterize the KMS states we also describe the ground states on $C^{*}(\mathcal{G})$.

Our work is organized in the following way: In Section 2 we review the construction of ultragraph $\mathrm{C}^{*}$-algebras via partial crossed products given in [13] and prove a few auxiliary results. In particular we give a simpler description of the basis of the topological (shift) space associated to an ultragraph in [13]. In Section 3 we study arbitrary ultragraphs $\mathcal{G}$ and show that there exists a correspondence between the KMS of the $\mathrm{C}^{*}$-algebra $C^{*}(\mathcal{G})$ and a subset of the states on the core subalgebra of $C^{*}(\mathcal{G})$. In order to use the results in [10], regarding KMS states of partial dynamical systems, in Section 4 we restrict ourselves to ultragraphs with no sinks that satisfy Condition (RFUM) and prove Theorem 4.3, which generalizes (for ultragraphs with no sinks) Theorem 4.1 in [6] (we remark that our methods to prove this result are in great part different from the methods in [6]). In Section 5 we describe ground states in terms of subsets of the states on an Abelian algebra, a subset of the regular Borel probability measure on the spectrum of the just mentioned Abelian algebra, and as a subset of the functions on the generalized vertices of $\mathcal{G}$. We end the paper in Section [6, where we show the existence of KMS for the ultragraph $\mathrm{C}^{*}$-algebra that is neither an Exel Laca nor a graph algebra and completely characterize the ground states on the aforementioned algebra.

\section{Ultragraph $\mathrm{C}^{*}$-algebras viewed as partial crossed products}

\section{$2.1 \quad$ Ultragraph $\mathrm{C}^{*}$-algebras}

In this short subsection we recall the definition of ultragraph $\mathrm{C}^{*}$-algebras and a few key results, as done in [22].

Definition 2.1 An ultragraph is a quadruple $\mathcal{G}=\left(G^{0}, \mathcal{G}^{1}, r, s\right)$ consisting of two countable sets $G^{0}, \mathcal{G}^{1}$, a map $s: \mathcal{G}^{1} \rightarrow G^{0}$, and a map $r: \mathcal{G}^{1} \rightarrow$ 
$P\left(G^{0}\right) \backslash\{\emptyset\}$, where $P\left(G^{0}\right)$ stands for the power set of $G^{0}$.

Before we define the $\mathrm{C}^{*}$-algebra associated to an ultragraph we need the following notion.

Definition 2.2 Let $\mathcal{G}$ be an ultragraph. Define $\mathcal{G}^{0}$ to be the smallest subset of $P\left(G^{0}\right)$ that contains $\{v\}$ for all $v \in G^{0}$, contains $r(e)$ for all $e \in \mathcal{G}^{1}$, and is closed under finite unions and nonempty finite intersections.

The following description of $\mathcal{G}^{0}$ is useful.

Lemma 2.3 [22, Lemma 2.12] If $\mathcal{G}$ is an ultragraph, then

$$
\begin{array}{r}
\mathcal{G}^{0}=\left\{\bigcap_{e \in X_{1}} r(e) \cup \ldots \cup \bigcap_{e \in X_{n}} r(e) \cup F: X_{1}, \ldots, X_{n} \text { are finite subsets of } \mathcal{G}^{1}\right. \\
\text { and } \left.F \text { is a finite subset of } G^{0}\right\} .
\end{array}
$$

Furthermore, $F$ may be chosen to be disjoint from $\bigcap_{e \in X_{1}} r(e) \cup \ldots \cup \bigcap_{e \in X_{n}} r(e)$.

Definition 2.4 Let $\mathcal{G}$ be an ultragraph. The ultragraph algebra $C^{*}(\mathcal{G})$ is the universal $C^{*}$-algebra generated by a family of partial isometries with orthogonal ranges $\left\{s_{e}: e \in \mathcal{G}^{1}\right\}$ and a family of projections $\left\{p_{A}: A \in \mathcal{G}^{0}\right\}$ satisfying

1. $p_{\emptyset}=0, p_{A} p_{B}=p_{A \cap B}, p_{A \cup B}=p_{A}+p_{B}-p_{A \cap B}$, for all $A, B \in \mathcal{G}^{0}$;

2. $s_{e}^{*} s_{e}=p_{r(e)}$, for all $e \in \mathcal{G}^{1}$;

3. $s_{e} s_{e}^{*} \leq p_{s(e)}$ for all $e \in \mathcal{G}^{1}$; and

4. $p_{v}=\sum_{s(e)=v} s_{e} s_{e}^{*}$ whenever $0<\left|s^{-1}(v)\right|<\infty$.

\subsection{Notation}

Next we set up notation that will be used throughout the paper. This agrees with notation introduced in [18] and [12].

Let $\mathcal{G}$ be an ultragraph. A finite path in $\mathcal{G}$ is either an element of $\mathcal{G}^{0}$ or a sequence of edges $e_{1} \ldots e_{k}$ in $\mathcal{G}^{1}$ where $s\left(e_{i+1}\right) \in r\left(e_{i}\right)$ for $1 \leq i \leq k$. If 
we write $\alpha=e_{1} \ldots e_{k}$, the length $|\alpha|$ of $\alpha$ is just $k$. The length $|A|$ of a path $A \in \mathcal{G}^{0}$ is zero. We define $r(\alpha)=r\left(e_{k}\right)$ and $s(\alpha)=s\left(e_{1}\right)$. For $A \in \mathcal{G}^{0}$, we set $r(A)=A=s(A)$. The set of finite paths in $\mathcal{G}$ is denoted by $\mathcal{G}^{*}$. An infinite path in $\mathcal{G}$ is an infinite sequence of edges $\gamma=e_{1} e_{2} \ldots$ in $\prod \mathcal{G}^{1}$, where $s\left(e_{i+1}\right) \in r\left(e_{i}\right)$ for all $i$. The set of infinite paths in $\mathcal{G}$ is denoted by $\mathfrak{p}^{\infty}$. The length $|\gamma|$ of $\gamma \in \mathfrak{p}^{\infty}$ is defined to be $\infty$. A vertex $v$ in $\mathcal{G}$ is called a sink if $\left|s^{-1}(v)\right|=0$ and is called an infinite emitter if $\left|s^{-1}(v)\right|=\infty$.

For $n \geq 1$, we define $\mathfrak{p}^{n}:=\left\{(\alpha, A): \alpha \in \mathcal{G}^{*},|\alpha|=n, A \in \mathcal{G}^{0}, A \subseteq r(\alpha)\right\}$. We specify that $(\alpha, A)=(\beta, B)$ if and only if $\alpha=\beta$ and $A=B$. We set $\mathfrak{p}^{0}:=\mathcal{G}^{0}$ and we let $\mathfrak{p}:=\coprod_{n \geq 0} \mathfrak{p}^{n}$. We embed the set of finite paths $\mathcal{G}^{*}$ in $\mathfrak{p}$ by sending $\alpha$ to $(\alpha, r(\alpha))$. We define the length of a pair $(\alpha, A),|(\alpha, A)|$, to be the length of $\alpha,|\alpha|$. We call $\mathfrak{p}$ the ultrapath space associated with $\mathcal{G}$ and the elements of $\mathfrak{p}$ are called ultrapaths. Each $A \in \mathcal{G}^{0}$ is regarded as an ultrapath of length zero and can be identified with the pair $(A, A)$. We may extend the range map $r$ and the source map $s$ to $\mathfrak{p}$ by the formulas, $r((\alpha, A))=A$, $s((\alpha, A))=s(\alpha)$ and $r(A)=s(A)=A$.

We concatenate elements in $\mathfrak{p}$ in the following way: If $x=(\alpha, A)$ and $y=(\beta, B)$, with $|x| \geq 1,|y| \geq 1$, then $x \cdot y$ is defined if and only if $s(\beta) \in A$, and in this case, $x \cdot y:=(\alpha \beta, B)$. Also we specify that:

$$
x \cdot y= \begin{cases}x \cap y & \text { if } x, y \in \mathcal{G}^{0} \text { and if } x \cap y \neq \emptyset \\ y & \text { if } x \in \mathcal{G}^{0},|y| \geq 1, \text { and if } x \cap s(y) \neq \emptyset \\ x_{y} & \text { if } y \in \mathcal{G}^{0},|x| \geq 1, \text { and if } r(x) \cap y \neq \emptyset\end{cases}
$$

where, if $x=(\alpha, A),|\alpha| \geq 1$ and if $y \in \mathcal{G}^{0}$, the expression $x_{y}$ is defined to be $(\alpha, A \cap y)$. Given $x, y \in \mathfrak{p}$, we say that $x$ has $y$ as an initial segment if $x=y \cdot x^{\prime}$, for some $x^{\prime} \in \mathfrak{p}$, with $s\left(x^{\prime}\right) \cap r(y) \neq \emptyset$.

We extend the source map $s$ to $\mathfrak{p}^{\infty}$, by defining $s(\gamma)=s\left(e_{1}\right)$, where $\gamma=e_{1} e_{2} \ldots$ We may concatenate pairs in $\mathfrak{p}$, with infinite paths in $\mathfrak{p}^{\infty}$ as follows. If $y=(\alpha, A) \in \mathfrak{p}$, and if $\gamma=e_{1} e_{2} \ldots \in \mathfrak{p}^{\infty}$ are such that $s(\gamma) \in r(y)=A$, then the expression $y \cdot \gamma$ is defined to be $\alpha \gamma=\alpha e_{1} e_{2} \ldots \in \mathfrak{p}^{\infty}$. If $y=A \in \mathcal{G}^{0}$, we define $y \cdot \gamma=A \cdot \gamma=\gamma$ whenever $s(\gamma) \in A$. Of course $y \cdot \gamma$ is not defined if $s(\gamma) \notin r(y)=A$. 
Remark 2.5 When no confusion arises we will omit the dot in the notation of concatenation defined above, so that $x \cdot y$ will be denoted by $x y$.

Definition 2.6 For each subset $A$ of $G^{0}$, let $\varepsilon(A)$ be the set $\left\{e \in \mathcal{G}^{1}: s(e) \in\right.$ $A\}$. We shall say that a set $A$ in $\mathcal{G}^{0}$ is an infinite emitter whenever $\varepsilon(A)$ is infinite.

\subsection{The shift space associated to an ultragraph}

Our goal in this subsection is two-fold. We recall the shift space $X$ associated to an ultragraph without sinks (as in [13]) and prove some new results about its topology.

The key concept in the definition of the shift space $X$ associated to $\mathcal{G}$ is that of minimal infinite emitters. We recall this below.

Definition 2.7 Let $\mathcal{G}$ be an ultragraph and $A \in \mathcal{G}^{0}$. We say that $A$ is a minimal infinite emitter if it is an infinite emitter that contains no proper subsets (in $\mathcal{G}^{0}$ ) that are infinite emitters. Equivalently, $A$ is a minimal infinite emitter if it is an infinite emitter and has the property that, if $B \in \mathcal{G}^{0}$ is an infinite emitter, and $B \subseteq A$, then $B=A$. For a finite path $\alpha$ in $\mathcal{G}$, we say that $A$ is a minimal infinite emitter in $r(\alpha)$ if $A$ is a minimal infinite emitter and $A \subseteq r(\alpha)$. We denote the set of all minimal infinite emitters in $r(\alpha)$ by $M_{\alpha}$.

For later use we recall the following Lemma.

Lemma 2.8 Let $x=(\alpha, A) \in \mathfrak{p}$ and suppose that $A$ is a minimal infinite emitter. If the cardinality of $A$ is finite then it is equal to one and, if the cardinality of $A$ is infinite, then $A=\bigcap_{e \in Y} r(e)$ for some finite set $Y \subseteq \mathcal{G}^{1}$.

Associated to an ultragraph with no sinks, we have the topological space $X=\mathfrak{p}^{\infty} \cup X_{\text {fin }}$, where

$X_{\text {fin }}=\left\{(\alpha, A) \in \mathfrak{p}:|\alpha| \geq 1\right.$ and $\left.A \in M_{\alpha}\right\} \cup\left\{(A, A) \in \mathcal{G}^{0}: A\right.$ is a minimal infinite emitter $\}$, 
and the topology has a basis given by the collection

$\left\{D_{(\beta, B)}:(\beta, B) \in \mathfrak{p},|\beta| \geq 1\right\} \cup\left\{D_{(\beta, B), F}:(\beta, B) \in X_{\text {fin }}, F \subset \varepsilon(B),|F|<\infty\right\}$,

where for each $(\beta, B) \in \mathfrak{p}$ we have that

$$
D_{(\beta, B)}=\left\{(\beta, A): A \subset B \text { and } A \in M_{\beta}\right\} \cup\left\{y \in X: y=\beta \gamma^{\prime}, s\left(\gamma^{\prime}\right) \in B\right\}
$$

and, for $(\beta, B) \in X_{\text {fin }}$ and $F$ a finite subset of $\varepsilon(B)$,

$$
D_{(\beta, B), F}=\{(\beta, B)\} \cup\left\{y \in X: y=\beta \gamma^{\prime}, \gamma_{1}^{\prime} \in \varepsilon(B) \backslash F\right\} .
$$

Remark 2.9 For every $(\beta, B) \in \mathfrak{p}$, we identify $D_{(\beta, B)}$ with $D_{(\beta, B), F}$, where $F=\emptyset$. Furtheremore, we call the basic elements of the topology of $X$ given above by cylinder sets.

It was shown in [13] that the above topological space is metrizable and there a description of convergence of sequences is given. Furthermore, it was shown that, under Condition (RFUM), the shift space $X$ has a basis of open, compact sets. We recall Condition (RFUM) below.

Condition (RFUM): For each edge $e \in \mathcal{G}^{1}$ its range can be written as

$$
r(e)=\bigcup_{n=1}^{k} A_{n}
$$

where $A_{n}$ is either a minimal infinite emitter or a single vertex.

Under Condition (RFUM) we have the following characterization of the range of edges in $\mathcal{G}$.

Lemma 2.10 Let $\mathcal{G}$ be an ultragraph with no sinks that satisfies Condition (RFUM). Then each $A \in \mathcal{G}^{0}$ can be written uniquely as $A=\bigcup_{n=1}^{k} A_{n}$, where there exists an unique $k$ such that $\left|A_{k}\right|<\infty$ and $\varepsilon\left(A_{k}\right)<\infty, A_{j}$ is a minimal infinite emitter for $j \neq k$, and $A_{j} \cap A_{k}=\emptyset$ for all $j \neq k$.

\section{Proof.}


The existence part follows directly from Condition (RFUM) and the description of $\mathcal{G}^{0}$ given in Lemma 2.3.

Suppose that $A=\cup_{i=1}^{n} A_{i}=\cup_{i=1}^{m} A_{i}^{\prime}$, where $\left|A_{k}\right|$ and $\left|A_{k}^{\prime}\right|<\infty, \varepsilon\left(A_{k}\right)$ and $\varepsilon\left(A_{k}^{\prime}\right)<\infty$, and $A_{j} \cap A_{k}=\emptyset$ and $A_{j}^{\prime} \cap A_{k}^{\prime}=\emptyset$ for all $j \neq k$.

Notice that if $A_{i} \in M_{r(e)}$ and $\left|A_{i}\right|=1$ then $A_{i} \subseteq A_{j}^{\prime}$ for some $j \neq k$. Since $\mathcal{G}$ has no sinks and $A_{j}^{\prime}$ is a minimal infinite emitter, it follows that $A_{i}=A_{j}^{\prime}$. Now, if $A_{i} \in M_{r(e)}$ and $\left|A_{i}\right|=\infty$, then there exists $j \neq k$ such that $\left|A_{i} \cap A_{j}^{\prime}\right|=\infty$. Since there are no sinks, $A_{i} \cap A_{j}^{\prime}$ is a infinite emitter. Then, since both $A_{i}$ and $A_{j}^{\prime}$ are minimal infinite emitters, it follows that $A_{i}=A_{j}^{\prime}$. Therefore $\cup_{i \neq k} A_{i}=\cup_{i \neq k} A_{i}^{\prime}$ and hence $A_{k}=A_{k}^{\prime}$.

Corollary 2.11 Under the hypothesis of the above lemma, let $(\beta, B) \in \mathfrak{p}$. Then $D_{(\beta, B)}$ can be written as a finite, disjoint union of elements of the collection of cylinder sets $\left\{D_{(\beta, B), F}:(\beta, B) \in X_{\text {fin }}, F \subset \varepsilon(B),|F|<\infty\right\}$, union with the the collection $\left\{D_{(\alpha, A)}:(\alpha, A) \in \mathfrak{p}\right.$ and $\left.\varepsilon(A)<\infty\right\}$.

\section{Proof.}

Let $(\beta, B) \in \mathfrak{p}$. By Lemma $\left[2.10\right.$ we have that $B=\bigcup_{n=1}^{k} A_{n}$, where there exists an unique $k$ such that $\left|A_{k}\right|<\infty$ and $\varepsilon\left(A_{k}\right)<\infty, A_{j}$ is a minimal infinite emitter for $j \neq k$, and $A_{j} \cap A_{k}=\emptyset$ for all $j \neq k$. Let $F_{i}=\left\{e \in \mathcal{G}^{1}\right.$ : $\left.s(e) \in \cup_{j \neq k}\left(A_{i} \cap A_{j}\right)\right\}$ for all $i \neq k$, and let $V=\left\{s(e): e \in \cup_{i \neq k} F_{i}\right\}$. Then

$$
D_{(\beta, B)}=\bigsqcup_{i \neq k} D_{\left(\beta, A_{i}\right), F_{i}} \bigsqcup_{v \in V} D_{(\beta,\{v\})} \bigsqcup_{v \in A_{k}} D_{(\beta,\{v\})}
$$

and the union is disjoint.

Remark 2.12 It follows from Corollary 2.11 that the collection os sets described in that corollary forms a basis for the topology in $X$. Notice that if $(\alpha, A) \in \mathfrak{p}$ and $\varepsilon(A)<\infty$, then $D_{(\alpha, A)}=\sqcup_{e \in \varepsilon(A)} D(\alpha e, r(e))$, and the union is disjoint. Furthermore, for $(\alpha, A) \in \mathfrak{p}$ with $\varepsilon(A)<\infty$, and $F \subseteq \varepsilon(A)$, we denote the disjoint union $\sqcup_{e \in \varepsilon(A) \backslash F} D_{(\alpha e, r(e))}$ by $D_{(\alpha, A), F}$. 
Next we prove a result that will be necessary in Section 4, in the construction of a measure associated to a state in $C_{0}(X)$.

Proposition 2.13 Let $\mathcal{G}$ be an ultragraph with no sinks that satisfies Condition (RFUM). Then the collection of cylinder sets $\left\{D_{(\beta, B), F}:(\beta, B) \in\right.$ $\left.X_{\text {fin }}, F \subset \varepsilon(B),|F|<\infty\right\}$, union with the the collection $\left\{D_{(\alpha, A)}:(\alpha, A) \in\right.$ $\mathfrak{p}$ and $\varepsilon(A)<\infty\}$, union with the empty set, forms a semi-ring.

\section{Proof.}

Let $S$ denote the collection $\left\{D_{(\beta, B), F}:(\beta, B) \in X_{\text {fin }}, F \subset \varepsilon(B),|F|<\right.$ $\infty\}$, union with the the collection $\left\{D_{(\alpha, A)}:(\alpha, A) \in \mathfrak{p}\right.$ and $\left.\varepsilon(A)<\infty\right\}$, union with the empty set.

Notice that the intersection of two sets in $S$ is again in $S$.

We have to prove that if $C, C_{1} \in S$ are such that $C_{1} \subseteq C$, then there exists a finite sequence $C_{2}, C_{3} \ldots C_{n} \in S$ such that $C$ is equal to the union $\sqcup_{i=1}^{n} C_{i}$ and the $C_{i}$ are disjoint.

Let $C \in S$. Suppose that $C=D_{(\beta, B), F}$, for some $(\beta, B) \in X_{f i n},|F|<\infty$, and let $C_{1} \subset C$ be such that $C \backslash C_{1} \neq \emptyset$.

Since $C_{1} \subseteq C$ we have that $C_{1}=D_{\left(\beta \beta^{\prime}, A\right), F}$ for some $\beta^{\prime}$ and $A$ (notice that $\beta^{\prime}=r(\beta)$ and $F=\emptyset$ are allowed).

Suppose that $C_{1}=D_{(\beta, A), F^{\prime}}$, for $(\beta, A) \in X_{\text {fin }}$. Then $|\varepsilon(A)|=\infty$ and hence $A=B$. Therefore $C_{1}=D_{(\beta, B), F^{\prime}}$. It follows that

$$
C \backslash C_{1}=\bigsqcup_{e \in F^{\prime} \backslash F} D_{(\beta e, r(e))},
$$

and, since by Corollary 2.11 each $D_{(\beta e, r(e))}$ is a disjoint union of elements in $S$, we get that $C \backslash C_{1}$ is a disjoint union of elements in $S$. If $C_{1}=D_{(\beta, A)}$, with $|\varepsilon(A)|<\infty$, then $C \backslash C_{1}=D_{(\beta, B), F \cup \varepsilon(A)}$.

Next assume that $C_{1}=D_{\left(\beta \beta^{\prime}, A\right), F^{\prime}}$ with $\left|\beta^{\prime}\right| \geq 1$, say $\beta^{\prime}=\beta_{1}^{\prime} \ldots \beta_{n}^{\prime}$. Suppose that $\left(\beta \beta^{\prime}, A\right) \in X_{\text {fin }}$. By Lemma 2.10 we can write $r\left(\beta^{\prime}\right)=A_{1} \cup$ $\ldots A_{k} \ldots A_{M}$, where $k$ is such that $\left|A_{k}\right|<\infty$ and $\varepsilon\left(A_{k}\right)<\infty, A_{j}$ is a minimal infinite emitter for $j \neq k$, and $A_{j} \cap A_{k}=\emptyset$ for all $j \neq k$. With this we obtain 
that

$$
\begin{gathered}
C \backslash C_{1}=D_{(\beta, B), F \cup\left\{\beta_{1}^{\prime}\right\}} \sqcup D_{\left(\beta \beta_{1}^{\prime}, r\left(\beta_{1}^{\prime}\right)\right),\left\{\beta_{2}^{\prime}\right\}} \sqcup \ldots \sqcup D_{\left(\beta \beta_{1}^{\prime} \ldots \beta_{n-1}^{\prime}, r\left(\beta_{n-1}^{\prime}\right)\right),\left\{\beta_{n}^{\prime}\right\}} \bigsqcup \\
\bigsqcup_{A_{i} \neq A} D_{\left(\beta \beta^{\prime}, A_{i}\right),\left\{\varepsilon\left(\left(\cup A \cap A_{i}\right)\right)\right\}} \bigsqcup_{e \in F^{\prime}} D_{\left(\beta \beta^{\prime} e, r(e)\right) .}
\end{gathered}
$$

Since by Corollary 2.11 each $D_{\left(\beta \beta^{\prime} e, r(e)\right)}$ can be written as a disjoint union of elements in $S$ we get the desired description of $C \backslash C_{1}$.

Now suppose that $C_{1}=D_{\left(\beta \beta^{\prime}, A\right)}$ with $|\varepsilon(A)|<\infty$. Using the same description of $r\left(\beta^{\prime}\right)$ as in the previous case we obtain that

$$
\begin{gathered}
C \backslash C_{1}=D_{(\beta, B), F \cup\left\{\beta_{1}^{\prime}\right\}} \sqcup D_{\left(\beta \beta_{1}^{\prime}, r\left(\beta_{1}^{\prime}\right)\right),\left\{\beta_{2}^{\prime}\right\}} \sqcup \ldots \sqcup D_{\left(\beta \beta_{1}^{\prime} \ldots \beta_{n-1}^{\prime}, r\left(\beta_{n-1}^{\prime}\right)\right),\left\{\beta_{n}^{\prime}\right\}} \bigsqcup \\
\bigsqcup_{i \neq k} D_{\left(\beta \beta^{\prime}, A_{i}\right),\{\varepsilon(A)\}} \bigsqcup_{e \in \varepsilon\left(A_{k} \backslash A\right)} D_{\left(\beta \beta^{\prime} e, r(e)\right)} .
\end{gathered}
$$

The case when $C=D_{(\beta, B)}$ with $|\varepsilon(B)|<\infty$ is done analogously.

\subsection{The crossed product construction of an ultragraph $\mathrm{C}^{*}$-algebra}

In this subsection we recall the construction of ultragraph $\mathrm{C}^{*}$-algebras as partial crossed products, as done in [13].

Let $\mathcal{G}$ be an ultragraph with no sinks that satisfy Condition (RFUM). Denote by $\mathbb{F}$ the free group generated by $\mathcal{G}^{1}$. We will define a partial action of $\mathbb{F}$ on $X$. For this, let $P \subset \mathbb{F}$ be defined by

$$
P:=\left\{e_{1} \ldots e_{n} \in \mathbb{F}: e_{i} \in \mathcal{G}^{1}: n \geq 1\right\}
$$

and define clopen sets $X_{c}$, for each $c \in \mathbb{F}$, as follows:

- for the neutral element $0 \in \mathbb{F}$ let $X_{0}=X$;

- for $a \in P$ define

$$
X_{a}=\left\{(\beta, B) \in X_{\text {fin }}: \beta_{1} \ldots \beta_{|a|}=a\right\} \cup\left\{\gamma \in \mathfrak{p}^{\infty}: \gamma_{1} \ldots \gamma_{|a|}=a\right\} \text {; and }
$$




$$
\begin{aligned}
& X_{a^{-1}}=\left\{(A, A) \in X_{\text {fin }}: A \subseteq r(a)\right\} \cup \\
& \cup\left\{(\beta, B) \in X_{\text {fin }}: s(\beta) \in r(a)\right\} \cup\left\{\gamma \in \mathfrak{p}^{\infty}: s(\gamma) \in r(a)\right\}
\end{aligned}
$$

- for $a, b \in P$ with $a b^{-1}$ in its reduced form, define

$$
\begin{aligned}
& X_{a b^{-1}}=\left\{(a, A) \in X_{\text {fin }}: A \subseteq r(a) \cap r(b)\right\} \cup \\
& \cup\left\{(\beta, B) \in X_{\text {fin }}: \beta_{1} \ldots \beta_{|a|}=a \text { and } s\left(\beta_{|a|+1}\right) \in r(a) \cap r(b)\right\} \cup \\
& \cup\left\{\gamma \in \mathfrak{p}^{\infty}: \gamma_{1} \ldots \gamma_{|a|}=a \text { and } s\left(\gamma_{|a|+1}\right) \in r(a) \cap r(b)\right\} ;
\end{aligned}
$$

- for all other $c \in \mathbb{F}$ define $X_{c}=\emptyset$.

Remark 2.14 Notice that if $a \in P$ is not a path then $X_{a}$ is empty. Analogously, if $a, b \in P$ and $r(a) \cap r(b)=\emptyset$ then $X_{a b^{-1}}$ is empty. It is proved in [13] that each $X_{c}$, with $c \in \mathbb{F}$, is clopen and compact. Furthermore, for each $A \in \mathcal{G}^{0}, X_{A}:=\{x \in X: s(x) \subseteq A\}$ is clopen and compact.

Next we recall the definition of the homeomorphisms between the nonempty sets $X_{c}, c \in \mathbb{F}$. For each $a \in P$ such that $X_{a}$ is non-empty, let $\theta_{a}: X_{a^{-1}} \rightarrow X_{a}$ be defined by $\theta_{a}(x)=a \cdot x$, for each $x \in X_{a^{-1}}$ (here we are using the embedding of $a$ in $\mathfrak{p}$ as $(a, r(a)))$. Let $\theta_{a^{-1}}: X_{a} \rightarrow X_{a^{-1}}$ be defined by $\theta_{a^{-1}}((a, A))=(A, A), \theta_{a^{-1}}(a b, B)=(b, B)$ and $\theta(a \gamma)=\gamma$. Finally, for $a, b \in P$ such that $X_{a b^{-1}}$ is non-empty, let $\theta_{a b^{-1}}: X_{b a^{-1}} \rightarrow X_{a b^{-1}}$ be defined by $\theta_{a b^{-1}}(x)=a \cdot \theta_{b^{-1}}(x)$.

Remark 2.15 Since, for each $t \in \mathbb{F}$, the map $\theta_{t}: X_{t^{-1}} \rightarrow X_{t}$ is a homeomorphism we get that $\alpha_{t}: C\left(X_{t^{-1}}\right) \rightarrow C\left(X_{t}\right)$, defined by $\alpha_{t}(f)=f \circ \theta_{t^{-1}}$, is $a{ }^{*}$-isomorphism. It follows that $\left(\left\{X_{t}, h_{t}\right)\right\}_{t \in \mathbb{F}}$ is a topological partial action and, consequently, $\left(\left\{C\left(X_{t}\right)\right\}_{t \in \mathbb{F}},\left\{\alpha_{t}\right\}_{t \in \mathbb{F}}\right)$ is a $C^{*}$-algebraic partial action of $\mathbb{F}$ in $C(X)$ (see for example [4]).

The following result is important for the partial crossed product description of $C^{*}(\mathcal{G})$ and for our work.

Lemma 2.16 Denote by $1_{A}$ the characteristic map of $X_{A}$, for $A \in \mathcal{G}^{0}$, and $1_{c}$ the characteristic map of $X_{c}$, for $c \in \mathbb{F}$. Then the subalgebra $D \subseteq C_{0}(X)$ generated by all the characteristic maps $1_{c}, 1_{A}$ and $\alpha_{c}\left(1_{c^{-1}} 1_{A}\right)$, with $c \in \mathcal{G}^{*}$ and $A \in \mathcal{G}^{0}$, is dense in $C_{0}(X)$. Moreover, for each $0 \neq g \in \mathbb{F}$, the subalgebra 
$D_{g}$ of $C\left(X_{g}\right)$ generated by all the maps $1_{g} 1_{c}, 1_{g} 1_{A}$ and $1_{g} \alpha_{c}\left(1_{c^{-1}} 1_{A}\right)$, with $c \in \bigcup_{n=1}^{\infty}\left(\mathcal{G}^{1}\right)^{n}$ and $A \in \mathcal{G}^{0}$, is dense in $C\left(X_{g}\right)$.

Remark 2.17 Notice that $1_{c}$ may be written as $\alpha_{c}\left(1_{c^{-1}} 1_{r(c)}\right)$. Furthermore, $\alpha_{c}\left(1_{c^{-1}} 1_{A}\right)=1_{r(c) \cap A} \circ \theta_{c^{-1}}$. So the subalgebra $D$ above can be seens as the algebra generated by the characteristic maps $1_{r(c) \cap A} \circ \theta_{c^{-1}}$, with $A \in \mathcal{G}^{0}$ and $c \in \mathcal{G}^{*}$.

Below we recall the isomorphism between $C^{*}(\mathcal{G})$ and $C_{0}(X) \rtimes_{\alpha} \mathbb{F}$.

Theorem 2.18 Let $\mathcal{G}$ be an ultragraph with no sinks that satisfies conditon (RFUM). Then there exists a bijective ${ }^{*}$-homomorphism $\Phi: C^{*}(\mathcal{G}) \rightarrow$ $C_{0}(X) \rtimes_{\alpha} \mathbb{F}$ such that $\Phi\left(s_{e}\right)=1_{e} \delta_{e}$, for each edge $e \in \mathcal{G}^{1}$, and $\Phi\left(p_{A}\right)=1_{A} \delta_{0}$, for all $A \in \mathcal{G}^{0}$.

We end this section describing the core algebra of $C^{*}(\mathcal{G})$ in partial crossed product terms.

Proposition 2.19 Let $\mathcal{G}$ be an ultragraph with no sinks that satisfies Condition (RFUM). Then $C_{0}(X)$ is *-isomorphic to the $C^{*}$-subalgebra of $C^{*}(\mathcal{G})$ generated by $\left\{s_{c} p_{A} s_{c}^{*} \mid A \in \mathcal{G}^{0}, c \in \mathcal{G}^{*}\right\}$, via a map that takes $1_{A}$ to $p_{A}$ and $\alpha_{c}\left(1_{c^{-1}} 1_{A}\right)$ to $s_{c} p_{A} s_{c}^{*}$, for every $A \in \mathcal{G}^{0}$, and every $c \in \mathcal{G}^{*}$ with $|c| \geq 1$.

\section{Proof.}

Recall that $C_{0}(X)$ is identified with $C_{0}(X) \delta_{0}$. By Lemma 2.16, and the subsequent Remark, the subalgebra $D \subseteq C_{0}(X)$ generated by all the maps of the form $\alpha_{c}\left(1_{c^{-1}} 1_{A}\right)$, with $c \in \mathcal{G}^{*}$ and $A \in \mathcal{G}^{0}$, is dense in $C_{0}(X)$. The result now follows since the map $\Phi^{-1}$ of Theorem 2.18 is a ${ }^{*}$-isomorphism (over its image) from $C_{0}(X) \delta_{0}$ into $C^{*}(\mathcal{G})^{\gamma}$, taking $1_{A} \delta_{0}$ to $p_{A}$ and $\alpha_{c}\left(1_{c^{-1}} 1_{A}\right) \delta_{0}=$ $1_{c} \delta_{c} 1_{A} \delta_{A} 1_{c^{-1}} \delta_{c^{-1}}$ to $s_{c} p_{A} s_{c}^{*}$, for every $A \in \mathcal{G}^{0}$ and $c \in \bigcup_{n=1}^{\infty}\left(\mathcal{G}^{1}\right)^{n}$. 


\section{KMS states of $C^{*}$-algebras associated to ar- bitrary ultragraphs}

In this section we describe the set of KMS states of certain one-parameter group of automorphisms of $C^{*}(\mathcal{G})$ in terms of states of the core algebra $C^{*}(\mathcal{G})^{\gamma}$. Throughout this section $\mathcal{G}$ is an arbitrary ultragraph.

\section{KMS and ground states.}

Suppose that $(A, \mathbb{R}, \rho)$ is a $C^{*}$-algebraic dynamical system. An element $a$ of $A$ is analytic if $t \mapsto \rho_{t}(a)$ is the restriction of an entire function $z \mapsto \rho_{z}(a)$ on $\mathbb{C}$. A state $\phi$ of $(A, \mathbb{R}, \rho)$ is a KMS state with inverse temperature $\beta$ (or a $\mathrm{KMS}_{\beta}$ state) if $\phi(a b)=\phi\left(b \rho_{i \beta}(a)\right)$ for all analytic elements $a, b$. It is usually enough to check the KMS condition on a set of analytic elements which span a dense subalgebra of $A$. A state $\phi$ on $A$ is a ground state of $(A, \mathbb{R}, \rho)$ if for every $a, b$ analytic in $A$, the entire function $z \mapsto \phi\left(a \rho_{z}(b)\right)$ is bounded on the upper-half plane.

\section{Generalized gauge action.}

Let $\mathcal{G}$ be an ultragraph and let $N$ be a positive function on $\mathcal{G}^{1}$ such that there is a constant $K$ such that $N(e)>K$ for all $e \in \mathcal{G}^{1}$. Extend the function $N$ to $N: \mathcal{G}^{*} \rightarrow \mathbb{R}_{+}^{*}$ by defining $N(A)=1$, for $A \in \mathcal{G}^{0}$, and $N(\lambda)=N\left(e_{1}\right) \ldots N\left(e_{m}\right)$ for $\lambda=e_{1} \ldots e_{m} \in \mathcal{G}^{*}$, with $|\lambda|>0$.

The following lemma shows that the function $N$ gives an action of $\mathbb{R}$ on $C^{*}(\mathcal{G})$.

Lemma 3.1 Let $\mathcal{G}$ be an ultragraph and let $N: \mathcal{G}^{1} \rightarrow \mathbb{R}_{+}^{*}$ as above. Then there is a strongly continuous action $\rho^{c}: \mathbb{R} \rightarrow \operatorname{Aut}\left(C^{*}(\mathcal{G})\right)$ such that $\rho_{t}^{c}\left(p_{A}\right)=$ $p_{A}$, for all $A \in \mathcal{G}^{0}$, and $\rho_{t}^{c}\left(s_{e}\right)=N(e)^{i t} s_{e}$ for $e \in \mathcal{G}^{1}$.

Proof. Fix $t \in \mathbb{R}$. For each $e \in \mathcal{G}^{1}$, define $T_{e}:=N(e)^{i t} s_{e}$. Since $T_{e} T_{e}^{*}=s_{e} s_{e}^{*}$ and $T_{e}^{*} T_{e}=s_{e}^{*} s_{e}$, it is easy to see that $\left\{T_{e}: e \in \mathcal{G}^{1}\right\}$ and $\left\{p_{A}: A \in \mathcal{G}^{0}\right\}$ satisfies the conditions of Definition 2.4. Now the universal property gives a 
homomorphism $\rho_{t}^{c}: C^{*}(\mathcal{G}) \rightarrow C^{*}(\mathcal{G})$ such that $\rho_{t}^{c}\left(p_{A}\right)=p_{A}$, for all $A \in \mathcal{G}^{0}$, and $\rho_{t}^{c}\left(s_{e}\right)=N(e)^{i t} s_{e}$ for $e \in \mathcal{G}^{1}$. Observe that $\rho_{t}^{c} \circ \rho_{t^{\prime}}^{c}=\rho_{t+t^{\prime}}^{c}$ for $t, t^{\prime} \in \mathbb{R}$. Also notice that the identity map on $C^{*}(\mathcal{G})$ is $\rho_{0}^{c}$. It follows that $\left(\rho_{t}^{c}\right)^{-1}=\rho_{-t}^{c}$ and hence $\rho_{t}^{c} \in \operatorname{Aut}\left(C^{*}(\mathcal{G})\right)$. Therefore $\rho^{c}$ is a homomorphism of $\mathbb{R}$ into the $\operatorname{Aut}\left(C^{*}(\mathcal{G})\right)$.

To see that $\rho^{c}$ is strongly continuous we must prove that $t \mapsto \rho_{t}^{c}(b)$ is continuous for all $b \in C^{*}(\mathcal{G})$. Fix $\epsilon>0$ and $b \in C^{*}(\mathcal{G})$. There is a linear combination $d$ of generators in $C^{*}(\mathcal{G})$ such that $\|b-d\|<\frac{\epsilon}{3}$. Since $t \mapsto \rho_{t}^{c}(d)$ is continuous, there exists some $\delta>0$ such that $|t-u|<\delta \Rightarrow\left\|\rho_{t}^{c}(d)-\rho_{u}^{c}(d)\right\|<$ $\frac{\epsilon}{3}$. Now for $|t-u|<\delta$ we have that

$$
\left\|\rho_{u}^{c}(b)-\rho_{t}^{c}(b)\right\| \leq\left\|\rho_{u}^{c}(b-d)\right\|+\left\|\rho_{u}^{c}(d)-\rho_{t}^{c}(d)\right\|+\left\|\rho_{t}^{c}(b-d)\right\|<\epsilon,
$$

as required.

\section{Some properties of $C^{*}(\mathcal{G})$.}

Given an ultragraph $\mathcal{G}$, it is shown in [22, page 351] that

$$
C^{*}(\mathcal{G})=\overline{\operatorname{span}}\left\{s_{\mu} p_{A} s_{\nu}^{*}: \mu, \nu \in \mathcal{G}^{*}, A \in \mathcal{G}^{0}\right\} .
$$

Now let $N: \mathcal{G}^{1} \rightarrow \mathbb{R}_{+}^{*}$ and let $\rho$ be the associated action as in Lemma 3.1. For every $s_{\mu} p_{A} s_{\nu}^{*} \in C^{*}(\mathcal{G})$, the map $t \mapsto \rho_{t}\left(s_{\mu} p_{A} s_{\nu}^{*}\right)=N(\mu)^{i t} N(\nu)^{-i t} s_{\mu} p_{A} s_{\nu}^{*}$ on $\mathbb{R}$ extends to an analytic function on all the complex plane. Thus there are analytic elements which span a dense subalgebra of $C^{*}(\mathcal{G})$, and therefore to study KMS states one can consider only these elements.

Also it is shown in [22, page 350] that for $\mu, \nu \in \mathcal{G}^{*}$, with $|\mu|,|\nu| \geq 1$, we have 


$$
s_{\nu}^{*} s_{\mu}= \begin{cases}s_{\nu^{\prime}}^{*} & \text { if } \nu=\mu \nu^{\prime}, \nu^{\prime} \notin \mathcal{G}^{0} \\ s_{\mu^{\prime}} & \text { if } \mu=\nu \mu^{\prime}, \mu^{\prime} \notin \mathcal{G}^{0} \\ p_{r(\nu)} & \text { if } \mu=\nu \\ 0 & \text { otherwise. }\end{cases}
$$

There is also a strongly continuous gauge action $\gamma: \mathbb{T} \rightarrow \operatorname{Aut}\left(C^{*}(\mathcal{G})\right)$ such that $\gamma_{z}\left(s_{\mu}\right)=z s_{\mu}$ and $\gamma_{z}\left(p_{A}\right)=p_{A}$. The core algebra $C^{*}(\mathcal{G})^{\gamma}$ is the fixed point subalgebra for the gauge action.

The proof of the next two lemmas works the same as for graph $\mathrm{C}^{*}$-algebras as done in Raeburn's book [20].

\section{Lemma 3.2}

$$
C^{*}(\mathcal{G})^{\gamma}=\overline{\operatorname{span}}\left\{s_{\mu} p_{A} s_{\nu}^{*}: \mu, \nu \in \mathcal{G}^{*}, A \in \mathcal{G}^{0},|\mu|=|\nu|\right\}
$$

Lemma 3.3 There is a conditional expectation $\Psi: C^{*}(\mathcal{G}) \rightarrow C^{*}(\mathcal{G})^{\gamma}$ such that

$$
\Psi\left(s_{\mu} p_{A} s_{\nu}^{*}\right)=\delta_{|\mu|,|\nu|} s_{\mu} p_{A} s_{\nu}^{*},
$$

for all $\mu, \nu \in \mathcal{G}^{*}, A \in \mathcal{G}^{0}$.

\section{KMS states for the generalized gauge action}

Lemma 3.4 Let $N: \mathcal{G}^{1} \rightarrow \mathbb{R}_{+}^{*}$ be such that $N(\mu) \neq 1$ for $\mu \in \mathcal{G}^{*} \backslash \mathcal{G}^{0}$ and let $\rho$ be the action of Lemma 3.1. Let $\beta \in \mathbb{R}$. Suppose that $\phi, \phi^{\prime}$ are $K M S_{\beta}$ states on $C^{*}(\mathcal{G})$ coinciding on the core algebra $C^{*}(\mathcal{G})^{\gamma}$. Then $\phi=\phi^{\prime}$.

Proof. Take an element $s_{\mu} p_{A} s_{\nu}^{*} \in C^{*}(\mathcal{G})$ with $|\mu| \neq|\nu|$ and let $\omega:=\phi-\phi^{\prime}$. We aim to show that $\omega\left(s_{\mu} p_{A} s_{\nu}\right)=0$. Since $\omega$ is a $\mathrm{KMS}_{\beta}$ state, by applying the 
KMS condition, we have that

$$
\begin{aligned}
\omega\left(s_{\mu} p_{A} s_{\nu}^{*}\right) & =\omega\left(p_{A} s_{\nu}^{*} \rho_{i \beta}\left(s_{\mu}\right)\right)=N(\mu)^{-\beta} \omega\left(p_{A} s_{\nu^{*}} s_{\mu}\right) \\
& = \begin{cases}N(\mu)^{-\beta} p_{A} s_{\mu^{\prime}} & \text { if } \mu=\nu \mu^{\prime}, \mu^{\prime} \notin \mathcal{G}^{0} \\
N(\mu)^{-\beta} p_{A} s_{\nu^{\prime}}^{*} & \text { if } \nu=\mu \nu^{\prime}, \nu^{\prime} \notin \mathcal{G}^{0} \\
0 & \text { otherwise. }\end{cases}
\end{aligned}
$$

Now it suffices to prove that $\omega\left(p_{B} s_{\lambda}\right)=\omega\left(p_{B} s_{\lambda}^{*}\right)=0$ for all $B \in \mathcal{G}^{0}, \lambda \in$ $\mathcal{G}^{*} \backslash \mathcal{G}^{0}$. To prove this, notice that if $C^{*}(\mathcal{G})$ has a unit, then

$$
\omega\left(p_{B} s_{\lambda}\right)=\omega\left(p_{B} s_{\lambda} 1\right)=N(\lambda)^{-\beta} \omega\left(1 p_{B} s_{\lambda}\right)=N(\lambda)^{-\beta} \omega\left(p_{B} s_{\lambda}\right) .
$$

Since $N(\lambda) \neq 1$, it follows that $\omega\left(p_{B} s_{\lambda}\right)=0$ as required.

If there is no unit for $C^{*}(\mathcal{G})$, we apply the same argument for the approximate unit $\left\{p_{A}: A \in \mathcal{G}^{0}\right\}$.

Proposition 3.5 Let $N: \mathcal{G}^{1} \rightarrow \mathbb{R}_{+}^{*}$ be such that $N(\mu) \neq 1$ for $\mu \in \mathcal{G}^{*} \backslash \mathcal{G}^{0}$ and let $\rho$ be the action of Lemma 3.1. Suppose that $\beta \in \mathbb{R}$ and $\phi$ is a state on $C^{*}(\mathcal{G})$. Then the restriction $\psi:=\left.\phi\right|_{C^{*}(\mathcal{G})^{\gamma}}$ satisfies

$$
\psi\left(s_{\mu} p_{A} s_{\nu}^{*}\right)=\delta_{\mu, \nu} N(\mu)^{-\beta} \psi\left(p_{A \cap r(\mu)}\right) .
$$

Conversely, for any state $\psi$ on $C^{*}(\mathcal{G})^{\gamma}$ satisfying (3), $\phi=\psi \circ \Psi$ is a $K M S_{\beta}$ state on $C^{*}(\mathcal{G})$, where $\Psi$ is the conditional expectation as in Lemma 3.3. Furthermore the obtained correspondence is an affine bijection.

Proof. Suppose that $\phi$ is a $\operatorname{KMS}_{\beta}$ state on $C^{*}(\mathcal{G})$ and let $\psi$ be its restriction to $C^{*}(\mathcal{G})^{\gamma}$. Take $s_{\mu} p_{A} s_{\nu} \in C^{*}(\mathcal{G})$ with $|\mu| \neq|\nu|$. Then the KMS condition impies that

$$
\phi\left(s_{\mu} p_{A} s_{\nu}^{*}\right)=\phi\left(p_{A} s_{\nu}^{*} \rho_{i \beta}\left(s_{\mu}\right)\right)=N(\mu)^{-\beta} \phi\left(p_{A} s_{\nu}^{*} s_{\mu}\right) .
$$

Therefore, by formula (2), we have that $\psi\left(s_{\mu} p_{A} s_{\nu}^{*}\right)=\delta_{\mu, \nu} N(\mu)^{-\beta} \psi\left(p_{A \cap r(\mu)}\right)$. 
Next suppose that $\psi$ is a state on $C^{*}(\mathcal{G})^{\gamma}$ which satisfies (3). To see that $\phi=\psi \circ \Psi$ is a $\mathrm{KMS}_{\beta}$ state, it suffices to prove the KMS condition

$$
\phi(a b)=N(\mu)^{-\beta} N(\nu)^{\beta} \phi(b a),
$$

for $a=s_{\mu} p_{A} s_{\nu}^{*}, b=s_{\lambda} p_{B} s_{\tau}^{*}$ where $\mu, \nu, \lambda, \tau \in \mathcal{G}^{*}$ and $A, B \in \mathcal{G}^{0}$. To see this first note that

$$
\begin{aligned}
a b=s_{\mu} p_{A} s_{\nu}^{*} s_{\lambda} p_{B} s_{\tau}^{*}= \begin{cases}s_{\mu} p_{A} s_{\nu^{\prime}}^{*} p_{B} s_{\tau}^{*} & \text { if } \nu=\lambda \nu^{\prime}, \nu^{\prime} \notin \mathcal{G}^{0} \\
s_{\mu} p_{A} s_{\lambda^{\prime}} p_{B} s_{\tau}^{*} & \text { if } \lambda=\nu \lambda^{\prime}, \lambda^{\prime} \notin \mathcal{G}^{0} \\
s_{\mu} p_{A \cap r(\nu) \cap B} s_{\tau}^{*} & \text { if } \lambda=\nu \\
0 & \text { otherwise. }\end{cases} \\
= \begin{cases}s_{\mu} p_{A} s_{\nu^{\prime}}^{*} s_{\tau}^{*} & \text { if } \nu=\lambda \nu^{\prime}, \nu^{\prime} \notin \mathcal{G}^{0}, s\left(\nu^{\prime}\right) \in B \\
s_{\mu} s_{\lambda^{\prime}} p_{B} s_{\tau}^{*} & \text { if } \lambda=\nu \lambda^{\prime}, \lambda^{\prime} \notin \mathcal{G}^{0}, s\left(\lambda^{\prime}\right) \in A \\
s_{\mu} p_{A \cap r(\nu) \cap B} s_{\tau}^{*} & \text { if } \lambda=\nu \\
0 & \text { otherwise. }\end{cases} \\
= \begin{cases}s_{\mu} p_{A} s_{\tau \nu^{\prime}} * & \text { if } \nu=\lambda \nu^{\prime}, \nu^{\prime} \notin \mathcal{G}^{0}, s\left(\nu^{\prime}\right) \in B \\
s_{\mu \lambda^{\prime}} p_{B} s_{\tau}^{*} & \text { if } \lambda=\nu \lambda^{\prime}, \lambda^{\prime} \notin \mathcal{G}^{0}, s\left(\lambda^{\prime}\right) \in A \\
s_{\mu} p_{A \cap r(\nu) \cap B} s_{\tau}^{*} & \text { if } \lambda=\nu \\
0 & \text { otherwise. }\end{cases}
\end{aligned}
$$

Similarly

$$
b a=s_{\lambda} p_{B} s_{\tau}^{*} s_{\mu} p_{A} s_{\nu}^{*}= \begin{cases}s_{\lambda} p_{B} s_{\nu \tau^{\prime}}^{*} & \text { if } \tau=\mu \tau^{\prime}, \tau^{\prime} \notin \mathcal{G}^{0}, s\left(\tau^{\prime}\right) \in A \\ s_{\lambda \mu^{\prime}} p_{A} s_{\nu}^{*} & \text { if } \mu=\tau \mu^{\prime}, \mu^{\prime} \notin \mathcal{G}^{0}, s\left(\mu^{\prime}\right) \in B \\ s_{\lambda} p_{B \cap r(\mu) \cap A^{\prime}} s_{\nu}^{*} & \text { if } \tau=\mu \\ 0 & \text { otherwise. }\end{cases}
$$


Now we apply $\phi=\psi \circ \Psi$ to both $a b$ and $b a$. Then

$$
\begin{gathered}
\phi(a b)= \begin{cases}N(\mu)^{-\beta} \psi\left(p_{A \cap r(\mu)}\right) & \text { if } \nu=\lambda \nu^{\prime}, \nu^{\prime} \notin \mathcal{G}^{0}, s\left(\nu^{\prime}\right) \in B, \mu=\tau \nu^{\prime} \\
N(\tau)^{-\beta} \psi\left(p_{B \cap r(\tau)}\right) & \text { if } \lambda=\nu \lambda^{\prime}, \lambda^{\prime} \notin \mathcal{G}^{0}, s\left(\lambda^{\prime}\right) \in A, \mu \lambda^{\prime}=\tau \\
N(\mu)^{-\beta} \psi\left(p_{A \cap r(\nu) \cap B \cap r(\mu)}\right) & \text { if } \lambda=\nu, \mu=\tau \\
0 & \text { otherwise, }\end{cases} \\
\phi(b a)= \begin{cases}N(\lambda)^{-\beta} \psi\left(p_{B \cap r(\lambda)}\right) & \text { if } \tau=\mu \tau^{\prime}, \tau^{\prime} \notin \mathcal{G}^{0}, s\left(\tau^{\prime}\right) \in A, \lambda=\nu \tau^{\prime} \\
N(\nu)^{-\beta} \psi\left(p_{A \cap r(\nu)}\right) & \text { if } \mu=\tau \mu^{\prime}, \mu^{\prime} \notin \mathcal{G}^{0}, s\left(\mu^{\prime}\right) \in B, \lambda \mu^{\prime}=\nu \\
N(\nu)^{-\beta} \psi\left(p_{B \cap r(\mu) \cap A \cap r(\nu)}\right) & \text { if } \tau=\mu, \lambda=\nu \\
0 & \text { otherwise. }\end{cases}
\end{gathered}
$$

Now if $\nu=\lambda \nu^{\prime}, s\left(\nu^{\prime}\right) \in B, \mu=\tau \nu^{\prime}$, then $r(\mu)=r\left(\nu^{\prime}\right)=r(\nu)$ and hence

$$
N(\mu)^{-\beta} N(\nu)^{\beta} \phi(b a)=N(\mu)^{-\beta} \psi\left(p_{A \cap r(\nu)}\right)=N(\mu)^{-\beta} \psi\left(p_{A \cap r(\mu)}\right)=\phi(a b) .
$$

If $\lambda=\nu \lambda^{\prime}, s\left(\lambda^{\prime}\right) \in A, \mu \lambda^{\prime}=\tau$, then $r(\tau)=r\left(\lambda^{\prime}\right)=r(\lambda)$. Also we have $\frac{N(\lambda)}{N(\nu)}=N\left(\lambda^{\prime}\right)=\frac{N(\tau)}{N(\mu)}$ and hence $N(\lambda)^{-\beta} N(\mu)^{-\beta} N(\nu)^{\beta}=N(\tau)^{-\beta}$. Then

$$
N(\mu)^{-\beta} N(\nu)^{\beta} \phi(b a)=N(\tau)^{-\beta} \psi\left(p_{B \cap r(\lambda)}\right)=N(\tau)^{-\beta} \psi\left(p_{A \cap r(\tau)}\right)=\phi(a b) .
$$

Finally if $\lambda=\nu, \mu=\tau$, then clearly

$$
N(\mu)^{-\beta} N(\nu)^{\beta} \phi(b a)=\phi(a b),
$$

and we have proven (4). 


\section{KMS states of ultragraph $\mathrm{C}^{*}$-algebras re- alized as partial crossed products}

In this section we will further describe the set of KMS states associated to the one-parameter group of automorphisms described in the previous section. To this end we will make use of the description of $\mathrm{C}^{*}$-algebras associated to ultragraphs that have no sinks and satisfy Condition (RFUM) as partial crossed products (see Section 2.4) and build from the ideas in Section 4 of [6]. Before we proceed we set up some notation and recall the construction of the one-parameter group of automorphisms via partial crossed product theory.

Assumption: From now on all ultragraphs are assumed to have no sinks.

Recall from [10, Theorem 4.3] that given any function $N: \mathcal{G}^{1} \rightarrow(1, \infty)$ there exists a unique strongly continuous one-parameter group $\sigma$ of automorphisms of $C_{0}(X) \rtimes \mathbb{F}$ such that

$$
\sigma_{t}(b)=(N(e))^{i t} b \text { and } \sigma_{t}(c)=c
$$

for all $e \in \mathcal{G}^{1}$, all $b \in C\left(X_{e}\right) \delta_{e}$, and all $c \in C_{0}(X) \delta_{0}$.

If $N(e)=\exp (1)$ for every $e \in E^{1}$, then $\sigma_{t}$ is $2 \pi$-periodic, and so induces a strongly continuous action $\beta: \mathbb{T} \rightarrow \operatorname{Aut}\left(C_{0}(X) \rtimes \mathbb{F}\right)$ such that $\beta_{z}\left(1_{e} \delta_{e}\right)=$ $z 1_{e} \delta_{e}$ and $\beta_{z}\left(f \delta_{0}\right)=f \delta_{0}$ for all $z \in \mathbb{T}, e \in \mathcal{G}^{1}$, and $f \in C_{0}(X)$. Alternatively, one can build such an action proceeding as in the proof of [13, Theorem 4.11].

We then have the following

Corollary 4.1 Let $\mathcal{G}$ be an ultragraph with no sinks that satisfies condition (RFUM), let $\gamma$ be the gauge action on $C^{*}(\mathcal{G}), \beta$ as above, and let $\Phi: C^{*}(\mathcal{G}) \rightarrow$ $C_{0}(X) \rtimes_{\alpha} \mathbb{F}$ be the isomorphism of Theorem [2.18. Then

$$
\Phi \circ \gamma_{z}=\beta_{z} \circ \Phi
$$

for all $z \in \mathbb{T}$.

Proceeding as in [6], given a function $N: \mathcal{G}^{1} \rightarrow(1, \infty)$, and letting $\sigma$ be the unique strongly continuous one-parameter group of automorphisms of 
$\mathbb{C}(X) \times \mathbb{F}$ given by $(\sqrt{5})$ we have, by the Corollary above and Theorem 2.18 , a unique strongly continuous one-parameter group $\sigma$ of automorphisms of $C^{*}(\mathcal{G})$ such that

$$
\sigma_{t}\left(s_{e}\right)=(N(e))^{i t} s_{e} \text { and } \sigma_{t}\left(p_{A}\right)=p_{A}
$$

for all $e \in \mathcal{G}^{1}$ and $A \in \mathcal{G}^{0}$.

Remark 4.2 In Section 3 we considered any positive function $N: \mathcal{G}^{1} \rightarrow \mathbb{R}_{*}^{+}$ such that $N(e)>K$ for some $K>0$. In this section we are restricting our attention to functions $N: \mathcal{G}^{1} \rightarrow(1, \infty)$. Furthermore, the one-parameter group of automorphisms considered above is of the same form as the oneparameter group of automorphisms considered in Lemma 3.1.

Next we set up some notation that will simplify our follow up statements.

For $0 \leq \beta<\infty$ we define the following sets:

$A^{\beta}$ : the set of $\mathrm{KMS}_{\beta}$ states for $C^{*}(\mathcal{G})$,

$B^{\beta}$ : the set of states $\omega$ of $C_{0}(X)$ that satisfy the scaling condition $\omega(f \circ$ $\left.\theta_{e}^{-1}\right)=N(e)^{-\beta} \omega(f)$ for all $e \in \mathcal{G}^{1}$ and all $f \in C_{0}\left(X_{e^{-1}}\right)$,

$C^{\beta}$ : the set of regular Borel probability measures $\mu$ on $X$ that satisfy the scaling condition $\mu\left(\theta_{e}(A)\right)=N(e)^{-\beta} \mu(A)$ for every $e \in \mathcal{G}^{1}$ and every Borel measurable subset $A$ of $X_{e^{-1}}$,

$D^{\beta}$ : the set of functions $m: \mathcal{G}^{0} \rightarrow[0,1]$ satisfying

m1. $\lim _{A \in \mathcal{G}^{0}} m(A)=1$;

m2. $m(A)=\sum_{e: s(e) \in A} N(e)^{-\beta} m(r(e))$ if $|\varepsilon(A)|<\infty$;

m3. $m(A) \geq \sum_{e \in F} N(e)^{-\beta} m(r(e))$ for every finite subset $F$ of $\varepsilon(A)$;

m4. $m(A \cup B)=m(A)+m(B)-m(A \cap B)$, and

$E^{\beta}$ : the set of states $\psi$ on $C^{*}(\mathcal{G})^{\gamma}$ satisfying $\psi\left(s_{c} p_{A} s_{d}^{*}\right)=\delta_{c, d} N(c)^{-\beta} \psi\left(p_{A \cap r(c)}\right)$, for all $c, d \in \mathcal{G}^{*}$ and $A \in \mathcal{G}^{0}$.

Our goal for the reminder of this section is to prove the following theorem. 
Theorem 4.3 Let $\mathcal{G}$ be an ultragraph with no sinks that satisfy Condition (RFUM). Then there exists a convex isomorphism between $A^{\beta}, B^{\beta}, C^{\beta}, D^{\beta}$, and $E^{\beta}$.

By Proposition 3.5 we already have a convex isomorphism between $A^{\beta}$ and $E^{\beta}$. To prove the other equivalences we need to prove a few auxiliary results first.

Proposition 4.4 Let $\mathcal{G}$ be an ultragraph with no sinks that satisfies Condition (RFUM) and let $M$ be a function from $\mathcal{G}^{1}$ to $[0,1]$. Then there is a convex, injective map between the set of states $\omega$ of $C_{0}(X)$ such that $\omega\left(f \circ \theta_{e}^{-1}\right)=M(e) \omega(f)$ for all $e \in \mathcal{G}^{1}$ and all $f \in C_{0}\left(X_{e^{-1}}\right)$, and the set of functions $m: \mathcal{G}^{0} \rightarrow[0,1]$ satisfying

$m 1$ '. $\lim _{A \in \mathcal{G}^{0}} m(A)=1$;

$m 2^{\prime} . m(A)=\sum_{e: s(e) \in A} M(e) m(r(e))$ if $|\varepsilon(A)|<\infty$;

m3'. $m(A) \geq \sum_{e \in F} M(e) m(r(e))$ for every finite subset $F$ of $\varepsilon(A)$;

$m 4^{\prime} . m(A \cup B)=m(A)+m(B)-m(A \cap B)$.

Furthermore, the correspondence takes a state $\omega$ to the function defined, for $A \in \mathcal{G}^{0}$, by $m(A)=\omega\left(1_{A}\right)$.

\section{Proof.}

Let $\omega$ be a state of $C_{0}(X)$ such that $\omega\left(f \circ \theta_{e}^{-1}\right)=M(e) \omega(f)$ for all $e \in \mathcal{G}^{1}$ and all $f \in C_{0}\left(X_{e^{-1}}\right)$. Let $m$ be the function from $\mathcal{G}^{0}$ to $[0,1]$ given by

$$
m(A)=\omega\left(1_{A}\right)
$$

First we prove ( $\left.{ }^{1} 1^{1}\right)$ : Notice that $\left\{A: A \in \mathcal{G}^{0}\right\}$ is a directed set (with inclusion as preorder), and $\left\{1_{A}: A \in \mathcal{G}^{0}\right\}$ is an increasing approximate unity for $C_{0}(X)$. Then, by Theorem 3.3 .3 of [19], we have that $1=\lim \omega\left(1_{A}\right)=$ $\lim m(A)$ and hence $m$ satisfies (m1).

Before we show (m2') and (m3') notice that if $e \in \mathcal{G}^{1}$, then

$$
\omega\left(1_{e}\right)=\omega\left(1_{e^{-1}} \circ \theta_{e}^{-1}\right)=M(e) \omega\left(1_{e^{-1}}\right)=M(e) \omega\left(1_{r(e)}\right)=M(e) m(r(e)) .
$$




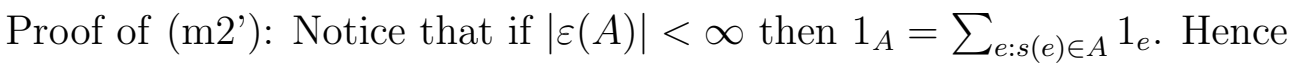

$$
m(A)=\omega\left(1_{A}\right)=\sum_{e: s(e) \in A} \omega\left(1_{e}\right)=\sum_{e: s(e) \in A} M(e) m(r(e))
$$

which gives (m2').

Proof of $(\underline{m 3})$ : Suppose that $F$ is a finite subset $\varepsilon(A)$. Then $1_{A} \geq$ $\sum_{e \in F} 1_{e}$ and we have that

$$
m(A)=\omega\left(1_{A}\right) \geq \sum_{e \in F} \omega\left(1_{e}\right)=\sum_{e \in F} M(e) m(r(e)) .
$$

Proof of (m4'): This follows from the linearity of $\omega$.

Next we prove that the correspondence given above is injective.

Let $\omega_{1}$ and $\omega_{2}$ be states such that $\omega_{i}\left(f \circ \theta_{e}^{-1}\right)=M(e) \omega_{i}(f)$ for all $e \in \mathcal{G}^{1}$ and all $f \in C_{0}\left(X_{e^{-1}}\right), i=1,2$. Suppose that $\omega_{1}\left(1_{A}\right)=\omega_{2}\left(1_{A}\right)$ for all $A \in \mathcal{G}^{0}$. We have to show that $\omega_{1}=\omega_{2}$. By Remark 2.17 it is enough to show that $\omega_{1}\left(1_{r(c) \cap A} \circ \theta_{c^{-1}}\right)=\omega_{2}\left(1_{r(c) \cap A} \circ \theta_{c^{-1}}\right)$ for all $A \in \mathcal{G}^{0}$ and $c \in \mathcal{G}^{*},|c| \geq 1$. This follows from the following computations for $c=c_{1} \ldots c_{n}$ and $A \in \mathcal{G}^{0}$ :

$$
\begin{aligned}
\omega_{1}\left(1_{r(c) \cap A} \circ \theta_{c_{n}^{-1} \ldots c_{1}^{-1}}\right) & =\omega_{1}\left(1_{\theta_{c_{n}}(r(c) \cap A)} \circ \theta_{c_{n-1}^{-1} \ldots c_{1}^{-1}}\right)=\ldots \\
& =\omega_{1}\left(1_{\theta_{c_{2} \ldots c_{n}}(r(c) \cap A)} \circ \theta_{c_{1}^{-1}}\right)=M\left(c_{1}\right) \omega_{1}\left(1_{\theta_{c_{2} \ldots c_{n}}}\right) \\
& =M\left(c_{1}\right) \omega_{1}\left(1_{\theta_{c_{3} \ldots c_{n}}} \circ \theta_{c_{2}}^{-1}\right)=\ldots \\
& =M\left(c_{1}\right) \ldots M\left(c_{n}\right) \omega_{1}\left(1_{r(c) \cap A}\right) \\
& =M\left(c_{1}\right) \ldots M\left(c_{n}\right) \omega_{2}\left(1_{r(c) \cap A}\right)=\ldots \\
& =\omega_{2}\left(1_{r(c) \cap A} \circ \theta_{c_{n}^{-1} \ldots c_{1}^{-1}}\right) .
\end{aligned}
$$

Next we will build a measure on the shift space $X$ following some of the ideas in section 5.5 of [8]. Recall that, as mentioned in Remark 2.9, we are writing $D_{(\alpha, A), \emptyset}$ for a cylinder of the form $D_{(\alpha, A)}$, so that all generalized cylinders can be written as $D_{(\beta, B), F}$. 
Given a function $M$ from $\mathcal{G}^{1}$ to $[0,1]$ and a function $m: \mathcal{G}^{0} \rightarrow[0,1]$ satisfying $\mathrm{m} 1$ '] to $\mathrm{m} 4$ ' of Proposition 4.4, we extend $M$ to $\mathcal{G}^{*}$ to the function $M: \mathcal{G}^{*} \rightarrow[0,1]$ given by $M(A)=m(A)$, for $A \in \mathcal{G}^{0}$, and $M(\beta)=$ $M\left(e_{1}\right) \ldots M\left(e_{n}\right)$ for $e_{1} \ldots e_{n} \in \mathcal{G}^{*}$, with $|\beta| \geq 1$. Now define a function on the set of all generalized cylinders by

$$
\kappa\left(D_{(\beta, B), F}\right)=M(\beta) m(B)-\sum_{e \in F} M(\beta e) m(r(e))
$$

which, by m3', takes values on non-negative numbers. We also define $\kappa(\emptyset)=$ 0 .

Lemma 4.5 Let $\mathcal{G}$ be an ultragraph with no sinks that satisfy Condition (RFUM) and $\kappa$ be the function defined by Equation (6). Then the restriction of $\kappa$ to the semi-ring $S$ given by Proposition 2.13 is a measure such that $\kappa\left(\theta_{e}(V)\right)=$ $M(e) \kappa(V)$, for every $e \in \mathcal{G}^{1}$ and every subset $V$ of $X_{e^{-1}} \cap S$.

\section{Proof.}

We have to prove that $\kappa$ is countably additive on $S$. Since all elements of $S$ are compact open sets, it is actually sufficient to show that $\kappa$ is additive on $S$. Suppose that

$$
D_{(\beta, B), F}=\bigsqcup_{i=1}^{n} D_{\left(\beta_{i}, B_{i}\right), F_{i}} .
$$

Notice that in this case, for all $i, \beta_{i}=\beta \beta_{i}^{\prime}$ for some $\beta_{i}^{\prime}$. We use an induction argument on $m=\max _{i}\left\{\left|\beta_{i}\right|-|\beta|\right\}$.

First suppose that $m=0$ so that $\beta_{i}=\beta$ and $B_{i} \subseteq B$ for all $i$. In the case that $(\beta, B) \in \mathfrak{p}$ with $|\varepsilon(\beta)|<\infty$, by our convention $F=\emptyset$. Also, since $B_{i} \subseteq B$, then $\left|\varepsilon\left(\beta_{i}\right)\right|<\infty$ and $F_{i}=\emptyset$ for all $i$. It follows that $B=\sqcup_{i} B_{i}$. By 
$\mathrm{m} 4$ '

$$
\begin{aligned}
\kappa\left(D_{(\beta, B)}\right) & =M(\beta) m(B) \\
& =M(\beta) m\left(\sqcup_{i} B_{i}\right) \\
& =M(\beta) m \sum_{i} m\left(B_{i}\right) \\
& =\sum_{i} \kappa\left(D_{\left(\beta_{i}, B_{i}\right)}\right) .
\end{aligned}
$$

For the case that $(\beta, B) \in X_{f i n}$, since $(\beta, B) \in D_{(\beta, B)}$, we must have $B_{i_{0}}=B$ for some $i_{0}$, and so $F_{i_{0}} \supseteq F$. For simplicity, suppose that $i_{0}=1$. Notice that for $i \neq 1, B_{i}$ cannot be a infinite emitter so that $F_{i}=\emptyset$. In order for (7) to hold, it is necessary that $\sqcup_{i \geq 2} \varepsilon\left(B_{i}\right)=F_{1} \backslash F$. Using the definition of $\kappa$ and $\mathrm{m} 2$ ' we obtain that

$$
\begin{aligned}
\kappa\left(D_{(\beta, B)}, F\right) & =M(\beta) m(B)-\sum_{e \in F} M(\beta e) m(r(e)) \\
& =M(\beta) m(B)-\sum_{e \in F_{1}} M(\beta e) m(r(e))+\sum_{e \in F_{1} \backslash F} M(\beta e) m(r(e)) \\
& =M(\beta) m(B)-\sum_{e \in F_{1}} M(\beta e) m(r(e))+\sum_{i=2}^{n} \sum_{e \in \varepsilon\left(B_{i}\right)} M(\beta e) m(r(e)) \\
& =M(\beta) m(B)-\sum_{e \in F_{1}} M(\beta e) m(r(e))+\sum_{i=2}^{n} M(\beta) m\left(B_{i}\right) \\
& =\sum_{i} \kappa\left(D_{\left(\beta_{i}, B_{i}\right), F_{i}}\right) .
\end{aligned}
$$

Now suppose that the result holds for all $0 \leq k<m$. Let us do the case where $(\beta, B) \in X_{\text {fin }}$, the other being analogous (CHECK). As above, we must have that there exists $i_{0}$ such that $\beta_{i_{0}}=\beta, B_{i_{0}}=B$ and $F_{i_{0}} \supseteq F$. Again, for simplicity, suppose that $i_{0}=1$. Also suppose that for some $i_{1}, \beta_{i}=\beta$ for $2 \leq i \leq i_{1}$ and $\beta_{i} \neq \beta$ for $i_{1}<i \leq n$. As in the case $m=0, F_{i}=\emptyset$ for $2 \leq i \leq i_{1}$, but now $\sqcup_{2 \leq i \leq i_{1}} \varepsilon\left(B_{i}\right) \subseteq F_{1} \backslash F$. Define 
$C=s\left(\left(F_{1} \backslash F\right) \backslash \sqcup_{2 \leq i \leq i_{1}} \varepsilon\left(B_{i}\right)\right)$ so that $\varepsilon(C)$ is finite. Notice that

$$
D_{(\beta, B), F}=D_{(\beta, C)} \sqcup \bigsqcup_{i=1}^{i_{1}} D_{\left(\beta_{i}, B_{i}\right), F_{i}}
$$

and

$$
D_{(\beta, C)}=\bigsqcup_{i=i_{1}+1}^{n} D_{\left(\beta_{i}, B_{i}\right), F_{i}} .
$$

Also, by the case $m=0$, we have that

$$
\kappa\left(D_{(\beta, B), F}\right)=\kappa\left(D_{(\beta, C)}\right)+\sum_{i=1}^{i_{1}} \kappa\left(D_{\left(\beta_{i}, B_{i}\right), F_{i}}\right) .
$$

Since $\varepsilon(C)$ is finite then $D_{(\beta, C)}=\bigsqcup_{s(e) \in C} D_{(\beta e, r(e))}$. Using Corollary 2.11, $\mathrm{m} 2$ ' and $\mathrm{m} 4$ ', we can write

$$
D_{(\beta, C)}=\bigsqcup_{j} D_{\left(\beta e_{j}, A_{j}\right)},
$$

where $D_{\left(\beta e_{j}, A_{j}\right)} \in S$, in such way that

$$
\kappa\left(D_{(\beta, C)}\right)=\sum_{j} \kappa\left(D_{\left(\beta e_{j}, A_{j}\right)}\right)
$$

Now

$$
D_{\left(\beta e_{j}, A_{j}\right)}=D_{\left(\beta e_{j}, A_{j}\right)} \cap D_{(\beta, C)}=\bigsqcup_{i=i_{1}+1}^{n} D_{\left(\beta e_{j}, A_{j}\right)} \cap D_{\left(\beta_{i}, B_{i}\right), F_{i}}
$$

and notice that $D_{\left(\beta e_{j}, A_{j}\right)} \cap D_{\left(\beta_{i}, B_{i}\right), F_{i}}=D_{\gamma_{j}, D_{j}}$ for some element of $S$ such that $\left|\gamma_{j}\right|=\left|\beta_{i}\right|$ (since $\left|\beta_{i}\right| \geq|\beta|+1=\left|\beta e_{j}\right|$ ). This implies that $\max _{j}\left\{\left|\gamma_{j}\right|-\left|\beta e_{i}\right|\right\}<$ $m$. By the induction hypothesis

$$
\kappa\left(D_{\left(\beta e_{j}, A_{j}\right)}\right)=\sum_{i=i_{1}+1}^{n} \kappa\left(D_{\left(\beta e_{j}, A_{j}\right)} \cap D_{\left(\beta_{i}, B_{i}\right), F_{i}}\right) .
$$


On the other hand, for $i_{1}+1 \leq i \leq n$,

$$
D_{\left(\beta_{i}, B_{i}\right), F_{i}}=D_{\left(\beta_{i}, B_{i}\right), F_{i}} \cap D_{\beta, C}=\bigsqcup_{j} D_{\left(\beta e_{j}, A_{j}\right)} \cap D_{\left(\beta_{i}, B_{i}\right), F_{i}}
$$

and using the case $m=0$ (even if some intersections are empty) we obtain that

$$
\kappa\left(D_{\left(\beta_{i}, B_{i}\right), F_{i}}\right)=\sum_{j} \kappa\left(D_{\left(\beta e_{j}, A_{j}\right)} \cap D_{\left(\beta_{i}, B_{i}\right), F_{i}}\right) .
$$

Putting equations (9), (10) and (11) together we arrive at

$$
\kappa\left(D_{(\beta, C)}\right)=\sum_{i=i_{1}+1}^{n} \kappa\left(D_{\left(\beta_{i}, B_{i}\right), F_{i}}\right),
$$

and, from (8), we get that $\kappa$ is a measure.

Finally, to prove the scaling condition, notice that if $V=D_{(\beta, B), F}$ is an element of $S$ contained in $X_{e^{-1}}$ then $\theta_{e}(V)=D_{(e \beta, B), F}$. Hence, from the definition of $\kappa$, and the multiplicativity of $M$, the result follows.

From the above lemma we obtain the following result.

Proposition 4.6 Let $\mathcal{G}$ be an ultragraph with no sinks that satisfy Condition (RFUM) and let $M$ be a function from $\mathcal{G}^{1}$ to $[0,1]$. Then there is a convex map between the set of functions $m: \mathcal{G}^{0} \rightarrow[0,1]$ satisfying $m 1$ to $m_{4} 1$ of Proposition 4.4 and the set of regular, Borel, probability measures $\mu$ on $X$ satisfying that $\mu\left(\theta_{e}(V)\right)=M(e) \mu(V)$, for all $e \in \mathcal{G}^{1}$ and all Borel measurable subsets $V$ of $X_{e}^{-1}$.

\section{Proof.}

Using Carathéodory's extension theorem, there is a measure $\mu$ defined on the $\sigma$-algebra generated by $S$. Since $S$ forms a countable basis, this is actually the Borel $\sigma$-algebra.

To prove that $\mu$ is probability, we first show that $\mu\left(D_{(A, A)}\right)=m(A)$ for all $A \in \mathcal{G}^{0}$. Using condition (RFUM), we can write $A=A_{1} \cup \cdots \cup A_{n}$, where $A_{1}, \ldots, A_{n-1}$ are minimal infinite emitters and $A_{n}$ is such that $\varepsilon\left(A_{n}\right)<\infty$. In particular, $D_{\left(A_{i}, A_{i}\right)} \in S$ for all $i=1, \ldots, n$. Using the inclusion-exclusion 
principle, we can write $\mu(A)$ as sum of $\pm \mu$ applied on $D_{\left(A_{1}, A_{1}\right)}, \ldots, D_{\left(A_{n}, A_{n}\right)}$ and their intersections which are of the form $D_{(B, B)}$ and still belongs to $S$. By definition, $\mu\left(D_{(B, B)}\right)=\kappa\left(D_{(B, B)}\right)=m(B)$, whenever $D_{(B, B)} \in S$. Now, using the inclusion-exclusion principle again together with (m4') we conclude that $\mu\left(D_{(A, A)}\right)=m(A)$.

Now, since $X=\bigcup_{A \in \mathcal{G}^{0}} D_{(A, A)}$, using condition (m1') we see that $\mu(X)=$ 1. This also implies that $\mu$ is regular because all finite Borel measures are regular.

From equation (6), a convex combination of functions $m$ is preserved when passing to measures $\kappa$ on $S$, and therefore to measures $\mu$ on $X$.

That $\mu$ satisfies the scaling condition follows from the fact $\theta_{e}$ is bijective, so that it preserves unions and intersections, and that the condition holds for elements in the semi-ring $S$ as in Lemma 4.5.

Proposition 4.7 Let $\mathcal{G}$ be an ultragraph with no sinks that satisfy Condition (RFUM). There is an affine bijection between $B^{\beta}$ and $E^{\beta}$.

\section{Proof.}

Let $\psi$ be a state on $C^{*}(\mathcal{G})^{\gamma}$ satisfying $\psi\left(s_{c} p_{A} s_{d}^{*}\right)=\delta_{c, d} N(c)^{-\beta} \psi\left(p_{A \cap r(c)}\right)$, for all $c \in \mathcal{G}^{*}$ and $A \in \mathcal{G}^{0}$. Define a state $\omega$ on $C_{0}(X)$ by $\psi \circ \Phi^{-1}$, where $\Phi$ is the isomorphism of Theorem 2.18 (see also Proposition 2.19).

We need to check the scaling condition. It is enough to check it in the dense subset of $C_{0}(X)$ given in Lemma 2.16 (see also Remark 2.17).

Let $e \in \mathcal{G}^{1}$. Notice that if $1_{A} \in C_{0}\left(X_{e^{-1}}\right)$ then $A \subseteq X_{e^{-1}}=X_{r(e)}$. Then

$$
\begin{aligned}
\omega\left(1_{A} \circ \theta_{e^{-1}}\right) & =w\left(1_{A \cap r(e)} \circ \theta_{e^{-1}}\right)=\psi\left(s_{e} p_{A} s_{e}^{*}\right)=N(e)^{-\beta} \psi\left(p_{A \cap r(e)}\right) \\
& =N(e)^{-\beta} \psi\left(\Phi^{-1}\left(1_{A \cap r(e)}\right)\right)=N(e)^{-\beta} \omega\left(1_{A \cap r(e)}\right) .
\end{aligned}
$$

Now let $c \in \mathcal{G}^{*}$ with $|c| \geq 1, A \in \mathcal{G}^{0}$, and suppose that $1_{r(c) \cap A} \circ \theta_{c^{-1}} \in$ $C_{0}\left(X_{e^{-1}}\right)$. Since $f \in C_{0}\left(X_{e^{-1}}\right)$ we have that $s(c) \in r(e)$ and hence $r(c)=$ $r(e c)$. Then

$\omega\left(1_{r(c) \cap A^{\circ}} \theta_{c^{-1}} \circ \theta_{e^{-1}}\right)=\omega\left(1_{r(e c) \cap A^{\circ}} \circ \theta_{(e c)^{-1}}\right)=\psi\left(s_{e c} p_{A} s_{e c}^{*}\right)=N(e c)^{-\beta} \psi\left(p_{A \cap r(e c)}\right)$ 


$$
=N(e)^{-\beta} N(c)^{-\beta} \psi\left(p_{A \cap r(c)}\right)=N(e)^{-\beta} \psi\left(s_{c} p_{A} s_{c}^{*}\right)=N(e)^{-\beta} \omega\left(1_{r(c) \cap A^{\circ}} \circ \theta_{c^{-1}}\right) .
$$

Hence the scaling condition is satisfied.

To finish the proof, notice that the inverse of the correspondence above is given by the following map: Given a state $\omega$ on $C_{0}(X)$ that satisfies the scaling condition $\omega\left(f \circ \theta_{e}^{-1}\right)=N(e)^{-\beta} \omega(f)$ for all $e \in \mathcal{G}^{1}$ and all $f \in C_{0}\left(X_{e^{-1}}\right)$, define $\psi$ as the state on $C^{*}(\mathcal{G})^{\gamma}$ such that $\psi\left(s_{c} p_{A} s_{d}^{*}\right)=\delta_{c, d} \omega \circ \Phi$. Notice that $\psi \in E^{\beta}$, since for $c=c_{1} \ldots c_{n} \in \mathcal{G}^{*}$ and $A \in \mathcal{G}^{0}$, we have that

$$
\begin{aligned}
\psi\left(s_{c} p_{A} s_{c}^{*}\right) & =\omega\left(1_{r(c) \cap A} \circ \theta_{c^{-1}}\right)=N\left(c_{1}\right)^{-\beta} \omega\left(1_{r(c) \cap A} \circ \theta_{c_{n}^{-1} \ldots c_{2}^{-1}}\right) \\
& =N\left(c_{1}\right)^{-\beta} N\left(c_{2}\right)^{-\beta} \omega\left(1_{r(c) \cap A} \circ \theta_{c_{n}^{-1} \ldots c_{3}^{-1}}\right)=\ldots \\
& =N\left(c_{1}\right)^{-\beta} N\left(c_{2}\right)^{-\beta} \ldots N\left(c_{n}\right)^{-\beta} \omega\left(1_{r(c) \cap A}\right)= \\
& =N(c)^{-\beta} \psi\left(p_{A \cap r(c)}\right) .
\end{aligned}
$$

The next proposition is basically the same as [6, Lemma 3.7 and Proposition 3.8]. We give the idea of the proof.

Proposition 4.8 Let $\mathcal{G}$ be an ultragraph with no sinks that satisfy Condition (RFUM). There is an affine bijection between $B^{\beta}$ and $C^{\beta}$.

\section{Proof.}

The Riesz representation Theorem gives the bijection between states $\omega$ on $C_{0}(X)$ and Borel regular probabilities $\mu$ on $X$. Restrict the corresponding measure $\mu$ to $X_{e^{-1}}$ and observe that $C_{0}\left(X_{e^{-1}}\right)$ is dense in $L^{1}\left(X_{e^{-1}}, \mu\right)$. If in one hand $\omega$ is in $B^{\beta}$, then the scaling condition holds for functions in $C_{0}\left(X_{e^{-1}}\right)$ and then extends to elements of $L^{1}\left(X_{e^{-1}}, \mu\right)$, in particular characteristic functions of measurable sets of $X_{e^{-1}}$. If on the other hand $\mu$ is in $C^{\beta}$, then the scaling condition holds for characteristic functions, which extends to elements of $L^{1}\left(X_{e^{-1}}, \mu\right)$, and in particular to functions in $C_{0}\left(X_{e^{-1}}\right)$.

Remark 4.9 We end the section with the proof of Theorem 4.3.

\section{Proof.}


Notice that from Proposition 3.5 we have an affine isomorphism between $A^{\beta}$ and $E^{\beta}$ ), from Proposition 4.7 we have an affine isomorphism between $E^{\beta}$ and $B^{\beta}$ ), and from Proposition 4.8 we have an affine isomorphism between $C^{\beta}$ and $B^{\beta}$ ).

Taking $M: \mathcal{G}^{1} \rightarrow[0,1]$ defined by $M(e)=(N(e))^{-\beta}$ on Proposition 4.4 we obtain an affine map from $B^{\beta}$ to $D^{\beta}$, and taking the same $M$ on Proposition 4.6 we obtain an affine map from $D^{\beta}$ to $C^{\beta}$. The result now follows from the fact that the maps from $B^{\beta}$ to $D^{\beta}$, from $D^{\beta}$ to $C^{\beta}$ and from $C^{\beta}$ to $B^{\beta}$ compose to the identity.

\section{$5 \quad$ Ground States}

In this section we apply some of our previous results to characterize the set of ground states on the $\mathrm{C}^{*}$-algebra of an ultragraph with no sinks that satisfy Condition (RFUM) (we recall the general definition of ground states in the beginning of Section 3).

Let $\mathcal{G}$ be an ultragraph with no sinks that satisfy Condition (RFUM). Define

$A^{g r}$ : the set of ground states in $C^{*}(\mathcal{G})$,

$B^{g r}$ : the set of states $\omega$ of $C_{0}(X)$ such that $\omega\left(1_{e}\right)=0$, for all $e \in \mathcal{G}^{1}$,

$C^{g r}$ : the set of regular Borel probability measures $\mu$ on $X$ such that $\mu(A)=$ 0 , for every $e \in \mathcal{G}^{1}$ and every Borel measurable subset $A$ of $X_{e}$,

$D^{g r}$ : the set of functions $m: \mathcal{G}^{0} \rightarrow[0,1]$ satisfying

m1. $\lim _{A \in \mathcal{G}^{0}} m(A)=1$

m2. $m(A)=0$ if $|\varepsilon(A)|<\infty$;

m3. $m(A \cup B)=m(A)+m(B)-m(A \cap B)$.

Next we prove the following: 
Theorem 5.1 Let $\mathcal{G}$ be an ultragraph with no sinks that satisfy Condition (RFUM). Then there exists an affine isomorphism between $A^{g r}, B^{g r}, C^{g r}$, and $D^{g r}$.

\section{Proof.}

The existence of an affine isomorphism between $A^{g r}$ and the set of states $\phi$ of $C_{0}(X)$ such that $\phi(f)=0$, for all $e \in \mathcal{G}^{1}$ and $f \in C\left(X_{e}\right)$ follows from Theorem 4.3 of [10]. Since $\phi$ is a state, and $1_{e}$ is a unit for $C\left(X_{e}\right)$, it follows that if $\phi\left(1_{e}\right)=0$ then $\phi(f)=0$ for all $f \in C\left(X_{e}\right)$. Therefore we have that $A^{g r}$ is isomorphic to $B^{g r}$, via an affine isomorphism.

As with KMS states, an affine isomorphism between $B^{g r}$ and $C^{g r}$ is obtained analogously to Proposition 4.8 in [6].

Finally, an affine isomorphism between $B^{g r}$ to $D^{g r}$ is obtained by application of Propositions 4.4 and 4.6 with $M(e)=0$ for all $e \in \mathcal{G}^{1}$, and proceeding as in the proof of Theorem 4.3 .

\section{Example}

Below we recall the construction, given in [21], of an ultragraph such that the associated $\mathrm{C}^{*}$-algebra is neither a graph $\mathrm{C}^{*}$-algebra nor an Exel-Laca algebra.

Definition 6.1 If $I$ is a countable set and $A$ is an $I \times I$ matrix with entries in $\{0,1\}$, then we may form the ultragraph $\mathcal{G}_{A}:=\left(G_{A}^{0}, \mathcal{G}_{A}^{1}, r, s\right)$ defined by $G_{A}^{0}:=\left\{v_{i}: i \in I\right\}, \mathcal{G}_{A}^{1}:=\left\{e_{i}: i \in I\right\}, s\left(e_{i}\right)=v_{i}$ for all $i \in I$, and $r\left(e_{i}\right)=\left\{v_{j}: A(i, j)=1\right\}$.

Let $A$ be the countably infinite matrix

$$
A=\left(\begin{array}{cccccccc}
1 & 0 & 0 & 1 & 1 & 1 & 1 & \\
0 & 1 & 0 & 1 & 1 & 1 & 1 \\
0 & 0 & 1 & 1 & 1 & 1 & 1 \\
1 & 0 & 0 & 1 & 0 & 0 & 0 & \\
0 & 1 & 0 & 0 & 1 & 0 & 0 & \cdots \\
0 & 0 & 1 & 0 & 0 & 1 & 0 & \\
0 & 0 & 0 & 1 & 0 & 0 & 1 & \\
& & \vdots & & & & \ddots
\end{array}\right) .
$$

For the matrix $A$ above, let $\mathcal{G}:=\left(G^{0}, \mathcal{G}^{1}, r, s\right)$ be the ultragraph $\mathcal{G}_{A}$ of Definition 6.1. We define an ultragraph $\mathcal{F}$ by adding a single vertex $\{w\}$ 
to $\mathcal{G}$ and a countable number of edges with source $w$ and range $G^{0}$. More precisely, we define $\mathcal{F}:=\left(F^{0}, \mathcal{F}^{1}, r, s\right)$ by

$$
F^{0}:=\{w\} \cup G^{0} \quad \mathcal{F}^{1}:=\left\{f_{i}\right\}_{i=1}^{\infty} \cup \mathcal{G}^{1}
$$

and we extend $r$ and $s$ to $\mathcal{F}^{1}$ by defining $s\left(f_{i}\right)=\{w\}$ and $r\left(f_{i}\right)=G^{0}$ for all $1 \leq i<\infty$.

Our goal is to define a suitable function $N$ for which we can find $m$ that satisfies conditions $\mathrm{m} 1$ to $\mathrm{m} 4$ defining the set $D^{\beta}$. Using Definition 6.1 for the matrix $A$ above, we conclude that the set $\mathcal{F}^{0}$ is composed by all finite sets of vertices, and finite sets union with the set $B:=\left\{v_{4}, v_{5}, \ldots\right\}$. In particular $F^{0}$ is an element of $\mathcal{F}^{0}$.

Now, if a function $m$ satisfying $\mathrm{m} 1$ to $\mathrm{m} 4$ is to exist, for a given $N$ and $\beta$, using $\mathrm{m} 1$ and $\mathrm{m} 4$, we must have that

$$
m(\{w\})+m\left(\left\{v_{1}\right\}\right)+m\left(\left\{v_{2}\right\}\right)+m\left(\left\{v_{3}\right\}\right)+m(B)=m\left(F^{0}\right)=1 .
$$

Also, for $i=1,2,3$, using conditions $\mathrm{m} 2$ and $\mathrm{m} 4$, it should hold that

$$
m\left(\left\{v_{i}\right\}\right)=N\left(e_{i}\right)^{-\beta}\left(m\left(\left\{v_{i}\right\}\right)+m(B)\right),
$$

so that we can describe $m\left(\left\{v_{i}\right\}\right)$, for $i=1,2,3$, depending on $N, \beta$ and $m(B)$ as

$$
m\left(\left\{v_{i}\right\}\right)=\frac{N\left(e_{i}\right)^{-\beta}}{1-N\left(e_{i}\right)^{-\beta}} m(B)
$$

For $i \geq 4$, we have that $r\left(e_{i}\right)=\left\{v_{i-3}, v_{i}\right\}$. Using an induction on each congruence modulo 3 , we see that $m\left(\left\{v_{i}\right\}\right)$ can be written depending on $N$, $\beta$ and $m(B)$ using the edges $e_{j}$ for $j$ in the congruence class modulo 3 up to $i$. For simplicity, let us assume that for all $1 \leq i<\infty, N\left(e_{i}\right)=d$ for a fixed number $d \in(1, \infty)$ and define $d_{\beta}=\frac{d^{-\beta}}{1-d^{-\beta}}$. Notice that $d_{\beta}$ goes to 0 as $\beta$ goes to infinity. We claim that for $1 \leq i<\infty$, writing $i=3 q+r$ with $r=1,2,3$, we have that

$$
m\left(\left\{v_{i}\right\}\right)=d_{\beta}^{q+1} m(B) .
$$

This is the case for $q=0$. Now suppose it is true for a fixed $r=1,2,3$ and 
a given $q \geq 0$. Then

$$
m\left(v_{3(q+1)+r}\right)=d^{-\beta}\left(m\left(v_{3(q+1)+r}\right)+m\left(v_{3 q+r}\right)\right)
$$

and hence

$$
m\left(v_{3(q+1)+r}\right)=d_{\beta} m\left(v_{3 q+r}\right)=d_{\beta}^{q+2} m(B)
$$

Using this one can show that, if

$$
\frac{6 d_{\beta}^{2}}{1-d_{\beta}^{2}} \leq 1
$$

then $\mathrm{m} 3$ holds for $B$ and any finite subset $F$ of $\varepsilon(B)$.

Now, choose a sequence $\left\{c_{i}\right\}_{i=1}^{\infty}$ with $c_{i} \in(1, \infty)$ and define $N\left(f_{i}\right)=c_{i}$. Using equation (12), we can show that for $m 3$ to be true for $\{w\}$, it is necessary that for all $k \in \mathbb{N}$,

$$
\frac{m(\{w\})}{1-m(\{w\})} \geq \sum_{i=1}^{k} c_{i}^{-\beta} .
$$

It follows that if $\beta$ is such that the series $\sum_{i=1}^{\infty} c_{i}^{-\beta}$ converges and that (14) holds, then for any value of $m(\{w\}) \in[0,1)$ such that

$$
\frac{m(\{w\})}{1-m(\{w\})} \geq \sum_{i=1}^{\infty} c_{i}^{-\beta}
$$

we can define $m(B)$ and $m\left(\left\{v_{i}\right\}\right)$ for $i \in \mathbb{N}$ using equations (13) and (14).

Also, independently of $N$ and $\beta$, the choice $m(\{w\})=1$ and $m(B)=$ $m\left(\left\{v_{i}\right\}\right)=0$ for $i \in \mathbb{N}$ always satisfies m1-m4.

In the case of ground states, a function $m: \mathcal{G}^{0} \rightarrow[0,1]$ is is $D^{g r}$ if, and only if, $m\left(\left\{v_{i}\right\}\right)=0$ for $i \in \mathbb{N}$, and $m(B)+m(\{w\})=1$. 


\section{Acknowledgements}

The authors would like to thank Zahra Afsar for valuable discussions regarding the present paper. In particular, the second author would like to thank Zahra for teaching him the theory of KMS states.

\section{References}

[1] Z. Afsar, A. an Huef, and I. Raeburn. KMS states on $C^{*}$-algebras associated to local homeomorphisms. Internat. J. Math., 25(8):1450066, $28 \mathrm{pp}, 2014$.

[2] Z. Afsar and A. Sims. KMS states on the $C^{*}$-algebras of fell bundles over groupoids. arXiv:1708.00629, 2017.

[3] A. an Huef, M. Laca, I. Raeburn, and A. Sims. KMS states on $C^{*}-$ algebras associated to higher-rank graphs. J. Funct. Anal., 266(1):265$283,2014$.

[4] V. M. Beuter and D. Gonçalves. Partial crossed products as equivalence relation algebras. Rocky Mountain J. Math., 46(1):85-104, 2016.

[5] O. Bratteli and D. W. Robinson. Operator algebras and quantum statistical mechanics. 2. Texts and Monographs in Physics. Springer-Verlag, Berlin, second edition, 1997. Equilibrium states. Models in quantum statistical mechanics.

[6] T. M. Carlsen and N. S. Larsen. Partial actions and KMS states on relative graph $C^{*}$-algebras. J. Funct. Anal., 271(8):2090-2132, 2016.

[7] G. G. de Castro and F. de L. Mortari. KMS states for the generalized gauge action on graph algebras. C. R. Math. Acad. Sci. Soc. R. Can., 36(4):114-128, 2014.

[8] G. Edgar. Measure, topology, and fractal geometry. Undergraduate Texts in Mathematics. Springer, New York, second edition, 2008. 
[9] M. Enomoto, M. Fujii, and Y. Watatani. KMS states for gauge action on $O_{A}$. Math. Japon., 29(4):607-619, 1984.

[10] R. Exel and M. Laca. Partial dynamical systems and the KMS condition. Comm. Math. Phys., 232(2):223-277, 2003.

[11] D. Gonçalves, H. Li, and D. Royer. Branching systems and general Cuntz-Krieger uniqueness theorem for ultragraph $C^{*}$-algebras. Internat. J. Math., 27(10):1650083, 26, 2016.

[12] D. Gonçalves and D. Royer. Ultragraphs and shift spaces over infinite alphabets. Bull. Sci. Math., 141(1):25-45, 2017.

[13] D. Gonçalves and D. Royer. Infinite alphabet edge shift spaces via ultragraphs and their $C^{*}$-algebras. Int. Math. Res. Not., to appear.

[14] M. Imanfar, A. Pourabbas, and H. Larki. The leavitt path algebras of ultragraphs. arXiv:1701.00323, 2017.

[15] T. Kajiwara and Y. Watatani. KMS states on finite-graph $C^{*}$-algebras. Kyushu J. Math., 67(1):83-104, 2013.

[16] T. Katsura, P. S. Muhly, A. Sims, and M. Tomforde. Graph algebras, Exel-Laca algebras, and ultragraph algebras coincide up to Morita equivalence. J. Reine Angew. Math., 640:135-165, 2010.

[17] A. Kumjian and J. Renault. KMS states on $C^{*}$-algebras associated to expansive maps. Proc. Amer. Math. Soc., 134(7):2067-2078, 2006.

[18] A. E. Marrero and P. S. Muhly. Groupoid and inverse semigroup presentations of ultragraph $C^{*}$-algebras. Semigroup Forum, 77(3):399-422, 2008 .

[19] G. J. Murphy. $C^{*}$-algebras and operator theory. Academic Press, Inc., Boston, MA, 1990.

[20] I. Raeburn. Graph algebras, volume 103 of CBMS Regional Conference Series in Mathematics. Published for the Conference Board of the 
Mathematical Sciences, Washington, DC; by the American Mathematical Society, Providence, RI, 2005.

[21] M. Tomforde. Simplicity of ultragraph algebras. Indiana Univ. Math. J., 52(4):901-925, 2003.

[22] M. Tomforde. A unified approach to Exel-Laca algebras and $C^{*}$-algebras associated to graphs. J. Operator Theory, 50(2):345-368, 2003.

Gilles Gonçalves de Castro, Departamento de Matemática, Universidade Federal de Santa Catarina, Florianópolis, 88040-900, Brazil.

Email: gilles.castro@ufsc.br

Daniel Gonçalves, Departamento de Matemática, Universidade Federal de Santa Catarina, Florianópolis, 88040-900, Brazil.

Email: daemig@gmail.com 